\title{
Design of main linac emittance tuning bumps for the Compact Linear Collider and the International Linear Collider
}

\author{
Peder Eliasson* \\ European Organization for Nuclear Research (CERN), CH-1211 Geneva 23, Switzerland \\ and Department of Physics and Astronomy, Uppsala University, Box 516, SE-75120 Uppsala, Sweden \\ Daniel Schulte \\ European Organization for Nuclear Research (CERN), CH-1211 Geneva 23, Switzerland
}

(Received 12 September 2007; published 22 January 2008)

\begin{abstract}
The installation of elements in the main linac of future linear colliders can only be done with a limited precision. The inevitable misalignments lead to unacceptable emittance growth. Beam-based alignment, e.g., one-to-one correction, dispersion free steering, or ballistic alignment, is necessary to reduce the emittance growth. In some cases, this is, however, not sufficient. For further reduction of the emittance growth, so-called emittance tuning bumps have to be used. A general strategy for the design of emittance tuning bumps has been developed and tested. Simulations suggest that the method can be conveniently used to understand the weaknesses of existing emittance tuning bumps and to significantly improve their performance in terms of, e.g., emittance reduction capability and convergence speed. An example of an application is the design of ten orthogonal knobs that, according to simulations, can reduce the normalized emittance growth in the Compact Linear Collider (CLIC) main linac from 23.8 to $0.34 \mathrm{~nm}$ with convergence within two iterations. Four orthogonal knobs have also been designed for the International Linear Collider (ILC). Simulations show that these knobs converge within a single iteration and reduce normalized emittance growth from 3.8 to $0.05 \mathrm{~nm}$.
\end{abstract}

DOI: 10.1103/PhysRevSTAB.11.011002

PACS numbers: $29.20 .-\mathrm{c}$

\section{INTRODUCTION}

For both the Compact Linear Collider (CLIC) [1] and the International Linear Collider (ILC) [2], it is of highest importance that emittance is preserved throughout the machine. Emittance growth leads to luminosity decrease and consequently to a lower event rate for physics experiments. One of the parts of a collider that contributes most to emittance growth is the main linac. The emittance growth in the linac is mainly caused by wakefields in misaligned accelerating structures and by dispersion due to misaligned quadrupoles.

For CLIC, the element misalignments will be kept at a reasonable level by an active prealignment system that offers a precision of $10 \mu \mathrm{m}[3,4]$. The accuracy of the survey system proposed for ILC is $200 \mu \mathrm{m}$ [5]. For both machines, the limited alignment precision leads to unacceptable emittance growth, and one or more of the beambased alignment methods described below have to be used. For the simulations presented in this paper, the misalignments remaining after prealignment were based on numbers from [6,7], see Table I. The beam position monitor (BPM) resolution was assumed to be $0.1 \mu \mathrm{m}$ for CLIC and $1 \mu \mathrm{m}$ for ILC. All CLIC results were obtained using the $1500 \mathrm{GeV}$ lattice described in [8]. For ILC a laser-straight (not following the earth curvature) lattice was designed to accelerate electrons from 15 to $250 \mathrm{GeV}$ using an accel-

\footnotetext{
*peder.eliasson@gmail.com
}

erating gradient of $31.5 \mathrm{MV} / \mathrm{m}$ and an $\mathrm{rf}$ frequency of $1.3 \mathrm{GHz}$. There were 24 accelerating structures per quadrupole, and the phase advance per focusing-defocusing (FODO) cell was $75^{\circ}$ and $60^{\circ}$ in the horizontal and vertical plane, respectively. Beam parameters for CLIC and ILC are presented in Table II. Observe that, throughout this paper, the term emittance refers to normalized emittance $\left(\epsilon_{N}\right)$ in the vertical plane.

The method that is foreseen to be used in order to reduce emittance growth after prealignment is beam-based alignment. Beam-based alignment is a collective term for a few different alignment schemes that have in common that the BPM readings along the machine are used to align elements. Examples of such methods include one-to-one steering, dispersion free steering (DFS) [9], and ballistic alignment [10]. In the simulations presented in this paper the first two will be used.

TABLE I. Root-mean-square element misalignments after prealignment.

\begin{tabular}{lcc}
\hline \hline \multicolumn{1}{c}{ Element } & $\begin{array}{c}\sigma \\
\text { (CLIC) }\end{array}$ & $\begin{array}{c}\sigma \\
\text { (ILC) }\end{array}$ \\
\hline Quadrupole displacement $[\mu \mathrm{m}]$ & 50 & 300 \\
Accelerating structure displacement $[\mu \mathrm{m}]$ & 10 & 300 \\
BPM displacement $[\mu \mathrm{m}]$ & 10 & 200 \\
Accelerating structure vertical angle $[\mu$ radian $]$ & 10 & 300 \\
Girder displacement $[\mu \mathrm{m}]$ & 0 & 200 \\
Girder angle $[\mu$ radian] & 0 & 20 \\
\hline \hline
\end{tabular}


TABLE II. CLIC and ILC beam parameters.

\begin{tabular}{lcr}
\hline \hline \multicolumn{1}{c}{ Parameter } & CLIC & ILC \\
\hline Initial energy $[\mathrm{GeV}]$ & 9 & 15 \\
Final energy $[\mathrm{GeV}]$ & 1500 & 250 \\
Initial emittance $[\mathrm{nm}]$ & 5 & 20 \\
Particles per bunch $\left[10^{9}\right]$ & 2.56 & 20 \\
Bunch length $[\mu \mathrm{m}]$ & 30.8 & 300 \\
\hline \hline
\end{tabular}

The least complicated beam-based alignment method is one-to-one steering. Each quadrupole is displaced in order to center the beam in the next downstream BPM. Since the BPMs are themselves misaligned and have a limited resolution, the obtained beam trajectory is not the ideal one, and in general further alignment is necessary. Even though one-to-one steering is insufficient on its own, it is often used as a first step to give the more elaborate alignment schemes a better starting point.

In this case, DFS was used to improve emittance after the initial one-to-one steering. During DFS, the nominal beam and one or more test beams of different energies are used to simultaneously optimize the nominal trajectory and to minimize dispersion (by minimizing trajectory differences). In other words, the aim is to minimize the target function

$$
\chi^{2}=w_{0} \sum_{i=1}^{n} y_{0, i}^{2}+\sum_{j=1}^{m} \sum_{i=1}^{n}\left(y_{j, i}-y_{0, i}\right)^{2}
$$

where $n$ and $m$ are the number of BPMs and test beams, respectively. The offset of beam $j$ in BPM $i$ is denoted $y_{j, i}$, where $j=0$ for the nominal beam. The weight $w_{0}$ specifies the relative importance of the nominal trajectory and the dispersion.

For CLIC, a realignment of the accelerating structures has to be carried out as a last step of the beam-based alignment. This alignment can be done with a precision of $10 \mu \mathrm{m}$ using BPMs incorporated into the structures. Several previous CLIC studies show that, in addition to beam-based alignment, emittance optimization using emittance tuning bumps is absolutely necessary to achieve acceptable emittance levels [11-13].

An example of how much emittance growth can be reduced with beam-based alignment for initial misalignments according to Table I is shown in Fig. 1. The presented result is the average emittance for 100 randomly misaligned CLIC main linacs. The emittance at the entrance of the linac is $5 \mathrm{~nm}$, and the goal is to keep the emittance growth $\Delta \epsilon_{N}$ along the linac below $5 \mathrm{~nm}$ (corresponding to a relative emittance growth of $100 \%$ ). As can be seen the emittance growth is a factor of 100 too large in case only one-to-one correction is used. By using DFS in addition, the performance is significantly improved, and then finally by realigning the accelerating structures, the emittance growth is reduced to $23.8 \mathrm{~nm}$. This remaining

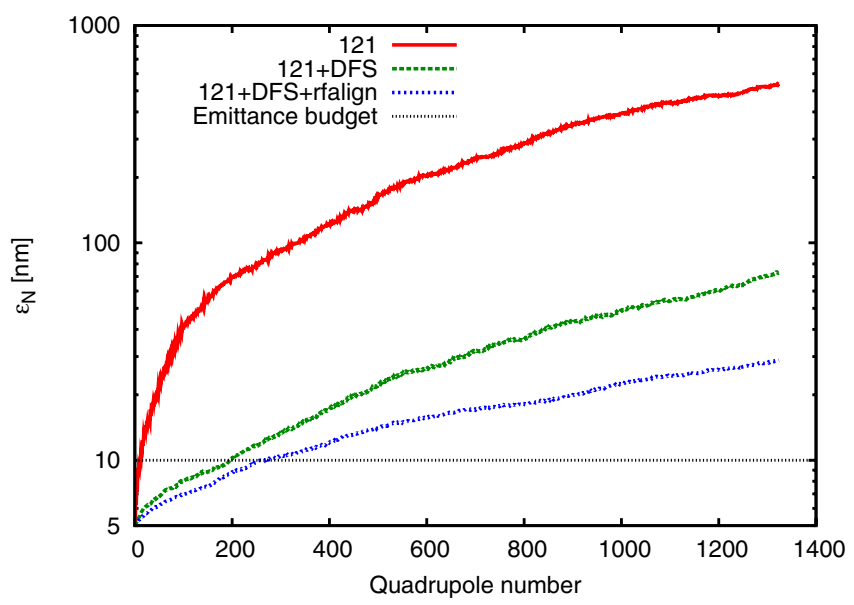

FIG. 1. Average emittance along 100 random CLIC main linacs for different beam-based alignment schemes. By using one-to-one (121) steering, DFS, and finally realigning the accelerating structures (rfalign), the emittance growth can be reduced to $23.8 \mathrm{~nm}$. To achieve the emittance growth target of $5 \mathrm{~nm}$, the additional use of tuning bumps is necessary.

emittance growth is far from acceptable and is mainly caused by wakefields. To reach the emittance goal, emittance tuning bumps have to be used.

For ILC, beam-based alignment alone might be sufficient to reach the emittance target [14]. The aim is that maximum $10 \%$ of the randomly misaligned ILC main linacs have a relative emittance growth of more than $50 \%$ $\left(\Delta \epsilon_{N}=10 \mathrm{~nm}\right)$. Despite the efficient beam-based alignment, emittance tuning bumps have been shown to reduce emittance growth significantly in ILC $[15,16]$.

The design of emittance tuning bumps is not trivial. The bumps should reduce emittance to acceptable levels, preferably very fast and in general under certain constraints, e.g., limited element displacements. In the following sections, the concept of emittance tuning bumps will be explained, a strategy for design of optimal bumps will be developed, and the new bumps will be shown to successfully reduce emittance growth to very low levels.

\section{EMITTANCE TUNING BUMPS}

\section{A. General description}

The emittance tuning bumps described here are based on the idea of "trajectory bumps" introduced in [17,18]. Examples of emittance tuning bumps include dispersion bumps and wakefield bumps [15].

A dispersion bump is used to introduce dispersion in $y$ and $y^{\prime}$ at some point along the linac. This may, for example, be achieved using displacements of two quadrupoles separated by an integer number of betatron oscillations. By kicking the beam out of its ideal orbit with the first quadrupole and back into the ideal orbit with the second one, dispersion is introduced. A problem is that the offset of the beam also generates wakefields in the accelerating struc- 
tures between the quadrupoles. By registering the effect on the emittance at the end of the linac, the dispersion setting that minimizes emittance can be found.

A wakefield bump, on the other hand, is most easily implemented as a displacement of one or more accelerating structures. If the structures are not on movers, wakefield bumps can also be constructed using quadrupole displacements, but in that case it is difficult to avoid introducing dispersion as well. The structure displacements are adjusted such that they minimize emittance at the end of the linac. Observe that in reality girders will be displaced rather than single accelerating structures. Each such girder supports a few accelerating structures.

The term emittance tuning bumps is, more generally, used to represent a set of so-called knobs and a measurement station. A knob is used to change some property of the beam line and consequently also of the beam. This property might be the vertical position of one or more accelerating structures (as for a wakefield bump) or the vertical position of a pair of quadrupoles (as for a dispersion bump). A knob might also be used to control, for example, BPM target values or to artificially introduce dispersion at a certain point along the beam line.

The purpose of the emittance tuning bumps is, as the name suggests, to minimize emittance. For the tuning bumps discussed in this paper, a station measuring the emittance at the end of the linac assures that the optimal knob settings are found. For CLIC, studies have also been carried out measuring emittance at several points along the linac [8]. In that case a knob was used to displace an accelerating structure in order to optimize the emittance locally, i.e., just after the structure. This was followed by an adjustment of the displacement of a downstream structure, once again to minimize emittance locally. A number of knobs could in this way be tuned, and since a change of one knob does not affect the upstream knobs and measure-

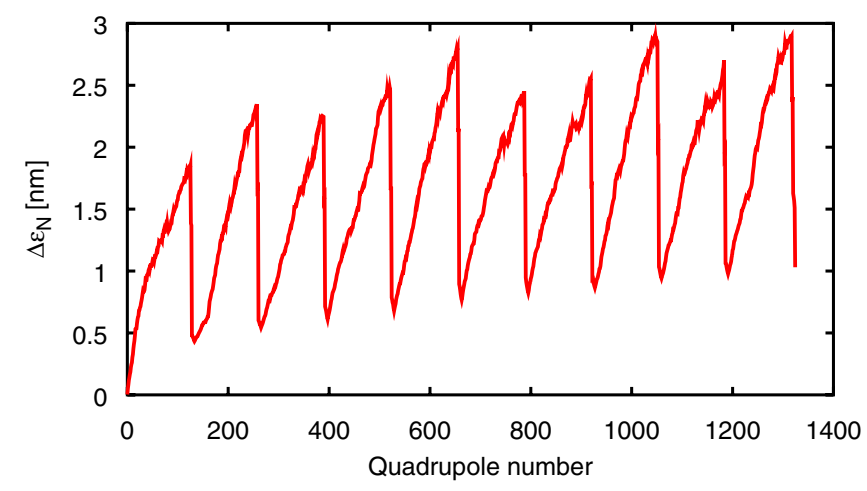

FIG. 2. Emittance growth along the CLIC main linac after beam-based alignment and tuning of 20 knobs each controlling the vertical displacement of an accelerating structure. The characteristic zigzag pattern is caused by two knobs canceling wakefields at each of ten positions along the linac. Between these bump positions emittance growth is not suppressed.

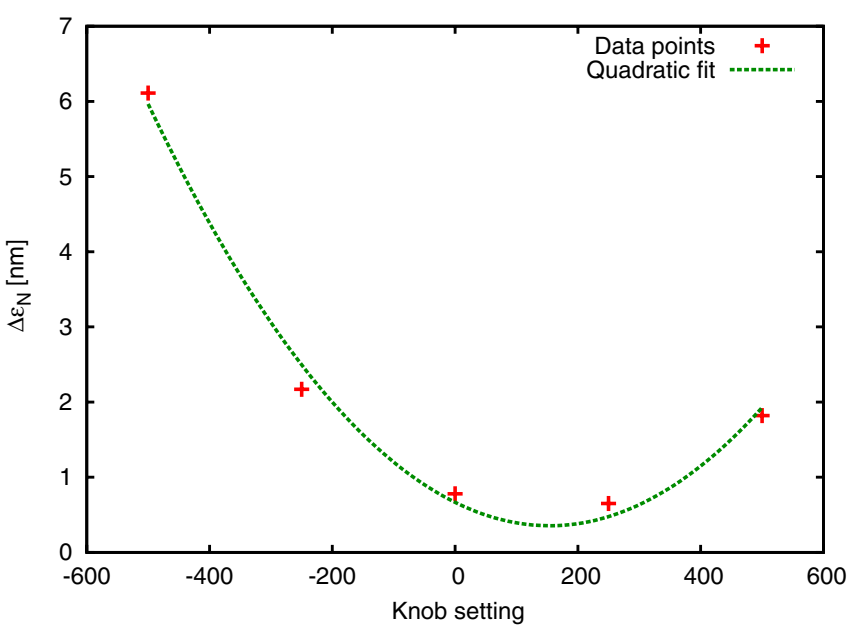

FIG. 3. The optimal knob setting is obtained by fitting a quadratic function to the emittance values measured for five different knob settings.

ment stations, no iterations are needed. The disadvantage of this method, apart from the obvious cost issue of using multiple measurement stations, is that local emittance minima do not guarantee that emittance is minimized globally, i.e., at the end of the linac. The performance of these bumps was still very good, and by displacing in total 20 accelerating structures, the emittance growth could be reduced to just over $1 \mathrm{~nm}$, see Fig. 2.

If emittance is instead measured only at the end of the linac, the knobs will no longer be independent, and several iterations may be needed to reach an optimum. This is of course a disadvantage, but at the same time these bumps are more powerful since they optimize emittance at the most relevant position. Besides, global emittance tuning bumps require only one measurement station.

The optimization method used to find the optimum knob settings is intentionally simple to make it usable not only during simulations but also in the control room. To tune a knob, five different settings are tested, and for each setting the measurement station reading is recorded. The optimum knob setting can then be determined with a quadratic fit to the obtained data points, see Fig. 3. Observe that emittance is approximately a quadratic function of the amplitude of an aberration. As long as the aberration amplitude is proportional to the knob setting, a quadratic function fits the data well. This is, for example, the case if a knob controls the displacements of quadrupoles or accelerating structures.

\section{B. Potential problems}

The performance of the tuning bumps is of course mainly determined by their capability to reduce emittance. However, several other factors also play an important role. One example is the amount of time required to minimize the emittance. This is related to the convergence speed of the knobs. 
As mentioned above, the bumps described in this paper are based on global emittance measurements. Since this in general means that the change of one knob setting affects the optimum of the others, iterations may be required for the knobs to converge, see Fig. 4. For strongly interfering knobs, the convergence may be very slow. In order to avoid this, the knobs have to be "decoupled," i.e., the change of one knob should no longer affect the optimum settings of any of the others. If the decoupling is successfully carried out, iterations will, as in the case of local emittance tuning bumps, be superfluous.

Another important issue to consider is the knob ranges. From a purely mathematical point of view, knob settings that reduce emittance to acceptable levels may exist. These optimal settings may, however, not lie within the range of the knobs. It is, for example, possible that the optimal settings require unacceptably large element displacements. Simulations show that the optimal accelerating structure displacements for CLIC emittance tuning are of the order of a mm when ten structures are used, see Fig. 5. In reality, displacements of this magnitude are unacceptable since it could lead to the beam hitting the beam pipe or the accelerating structure itself. Besides, the computer model of the accelerating structure does not take quadrupolar wakefields into account. Consequently, wakefield kicks are not accurately calculated for large beam offsets, and the obtained structure displacements will most likely not represent a real optimum. The most straightforward way to reduce the required displacements is to use a larger number of elements. By moving two adjacent accelerating structures together, the displacement needed can be reduced by a factor of 2 .

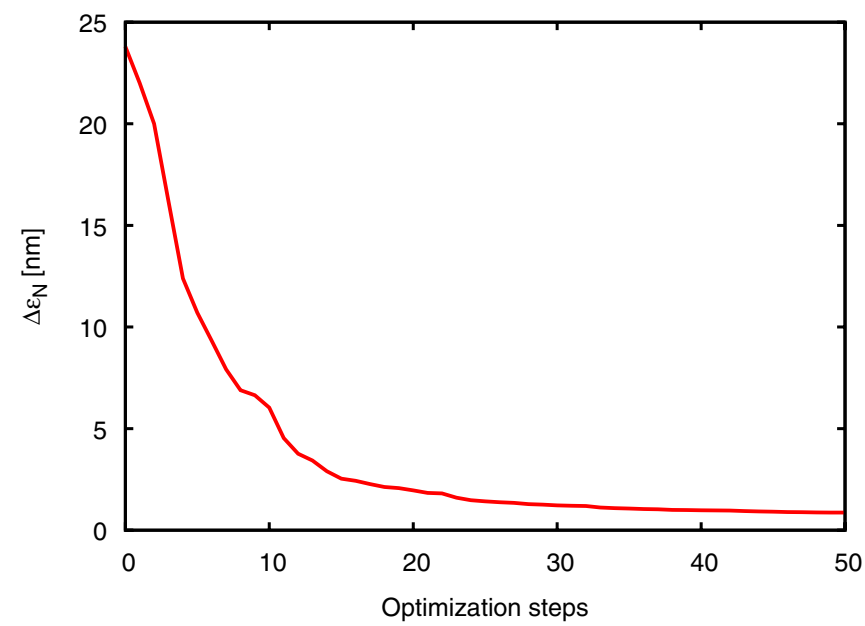

FIG. 4. Average emittance growth for 100 CLIC main linacs. The emittance growth is seen to decrease for each optimization step. Such an optimization step corresponds to the tuning of a single knob. In total ten knobs, each controlling the vertical displacement of an accelerating structure in the CLIC main linac, were used. Three or more iterations of the ten knobs seem to be needed for convergence.

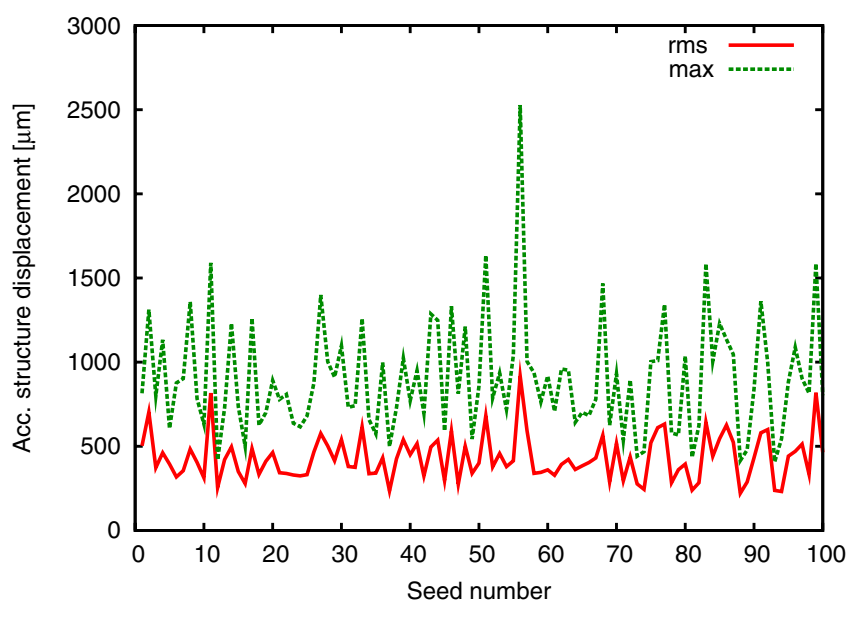

FIG. 5. Root-mean-square and maximum vertical displacement of ten accelerating structures used for CLIC emittance tuning. The results for 100 random machines are presented. The displacements should not be larger than a few tens of $\mu \mathrm{m}$.

If a knob is used to move an element, the minimum step size of the mover that the element is attached to might influence the performance of the tuning. In this paper, limited step size was modeled by only allowing the mover position to assume discrete values with a certain spacing (step size). The sensitivity to minimum step size increase with the number of elements moved. The solution is to limit the number of elements by identifying and using only the most efficient ones. Since the ILC quadrupoles may not be on movers, an alternative, nearly equivalent, solution could be to excite dipole correctors attached to the quadrupoles. This way of implementing the bumps, and the imperfections related to it, will not be studied here.

In the following two sections, describing the design and application of optimal bumps, the problem of convergence speed, knob range, and mover step size will be discussed in more detail. An issue that is not considered during the bump design is the limited resolution of the emittance measurement station. Previous simulations indicated that imperfections in the measurement station limit the performance of the bumps. It was, however, also observed that the effect can be reduced by modifying the optimization method used, see [15].

\section{DESIGN OF TUNING BUMPS}

The bump design described in this section takes its starting point in the macroparticle beam model used in many tracking codes, e.g., PLACET [19]. In this model, a beam is represented by a number of so-called macroparticles. Macroparticle $k$ is described by its energy and longitudinal position, horizontal position and angle, vertical position $\left(y_{k}\right)$ and angle $\left(y_{k}^{\prime}\right)$, its second momenta $\left(\sigma_{y y, k}, \sigma_{y y^{\prime}, k}, \sigma_{y^{\prime} y^{\prime}, k}\right)$, and finally its weight $\left(w_{k}\right)$.

The tuning bumps are assumed to be linear, i.e., the macroparticle positions and angles at the end of the ma- 
chine are linear with respect to the knob settings, and the second momenta are constant. Such bumps can be efficiently used to reduce emittance growth caused by e.g. dispersion and wakefield effects but may not be used to reduce emittance growth due to e.g. $x-y$ coupling.

Only vertical emittance will be considered and the beam at the end of the linac will be represented by a column vector

$$
\tilde{\mathbf{y}}=\left(y_{1}, y_{2}, \ldots, y_{p}, y_{1}^{\prime}, y_{2}^{\prime}, \ldots, y_{p}^{\prime}\right)^{T},
$$

where $y_{i}$ and $y_{i}^{\prime}$ are the position and angle of macroparticle $i$. A machine (seed), which has to be corrected using emittance tuning bumps, can similarly be represented by the macroparticle coordinates at the end of the machine:

$$
\tilde{\mathbf{s}}_{i}=\left(y_{1, i}, y_{2, i}, \ldots, y_{p, i}, y_{1, i}^{\prime}, y_{2, i}^{\prime}, \ldots, y_{p, i}^{\prime}\right)^{T} .
$$

Here the index $i$ is used to distinguish between different seeds. Knob $j$ may in a similar fashion be described by the coordinate changes caused by a unit change of the knob:

$$
\tilde{\mathbf{k}}_{j}=\left(\Delta y_{1, j}, \ldots, \Delta y_{p, j}, \Delta y_{1, j}^{\prime}, \ldots, \Delta y_{p, j}^{\prime}\right)^{T} .
$$

In other words, $\tilde{\mathbf{s}}_{i}$ contains the $p$ macroparticle positions and $p$ macroparticle angles at the end of machine $i$, while $\tilde{\mathbf{k}}_{j}$ contains the changes in macroparticle positions and angles for a unit change of knob $j$. A typical seed vector is visualized in Fig. 6, and two examples of knob vectors are depicted in Fig. 7. In both figures, the angle coordinates $y_{i}^{\prime}$ have been scaled by the Twiss parameter $\beta$ to obtain similar magnitudes for positions and angles.

The matrices $\tilde{\mathbf{S}}$ and $\tilde{\mathbf{K}}$ will be used to denote the collection of seeds and knobs, respectively, i.e.

$$
\tilde{\mathbf{S}}=\left(\tilde{\mathbf{s}}_{1}, \tilde{\mathbf{s}}_{2}, \ldots, \tilde{\mathbf{s}}_{n}\right)
$$

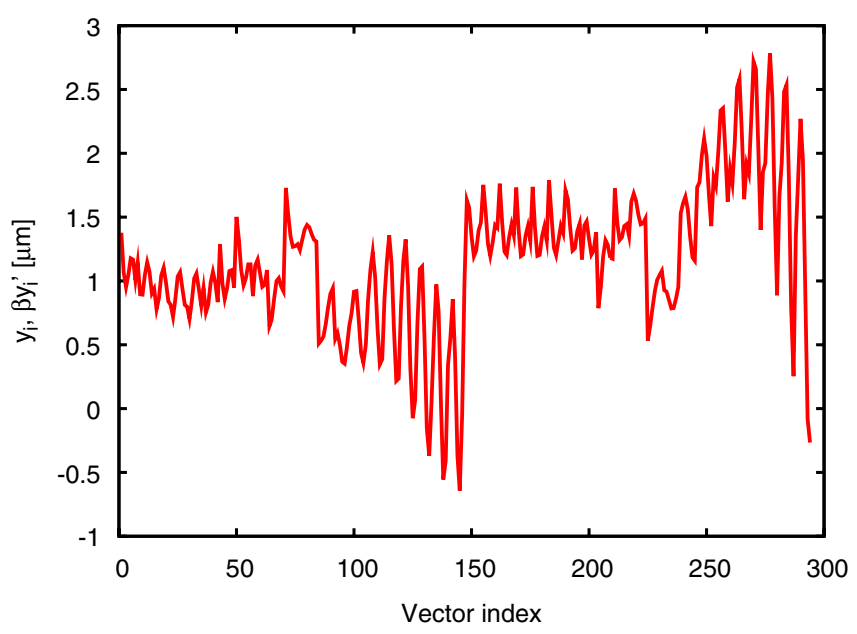

FIG. 6. Typical seed vector for a beam consisting of 147 macroparticles. Vector indices 1-147 represent macroparticle positions $\mathbf{y}_{i}$ and indices 148-294 represent scaled macroparticle angles $\beta \mathbf{y}_{i}^{\prime}$. For positions and angles, respectively, indices are ordered according to longitudinal position.

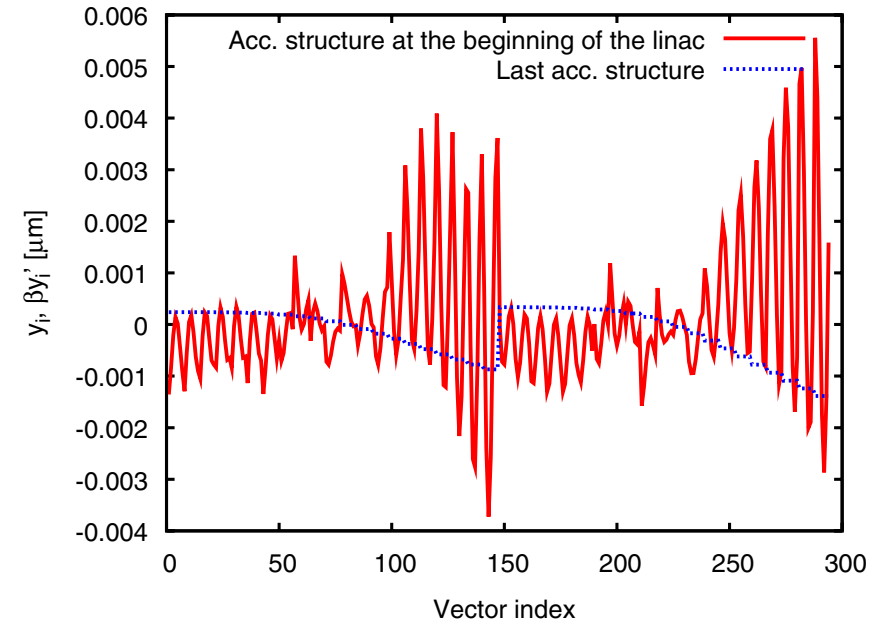

FIG. 7. Two knob vectors each corresponding to the displacement of an accelerating structure by $1 \mu \mathrm{m}$. Observe that for the last accelerating structure the characteristic wakefield kick is clearly visible (same index convention as in Fig. 6).

$$
\tilde{\mathbf{K}}=\left(\tilde{\mathbf{k}}_{1}, \tilde{\mathbf{k}}_{2}, \ldots, \tilde{\mathbf{k}}_{m}\right)
$$

If the seed vector $\tilde{\mathbf{s}}_{i}$ is in the range of the knob matrix $\tilde{\mathbf{K}}$, knob settings $\mathbf{x}_{i}$ exist such that

$$
\tilde{\mathbf{y}}=\tilde{\mathbf{s}}_{i}+\tilde{\mathbf{K}} \mathbf{x}_{i}=0
$$

These knob settings turn the seed vector, if depicted as in Fig. 6, into a straight line and the emittance is consequently minimized (leaving only the emittance $\epsilon_{N 0}$ associated with the second momenta of the macroparticles). Observe, however, that if no exact solution exists and a least-square solution is calculated instead, the emittance is not necessarily minimized. One of the reasons for this is that there is no direct correlation between minimized $|\tilde{\mathbf{y}}|^{2}$ and minimized emittance. The other reason is that Eq. (7) is not the only equation corresponding to minimized emittance. Any knob settings solving $y_{k}=A, y_{k}^{\prime}=B$ for all $k$ ( $A$ and $B$ being arbitrary constants) minimize emittance. If only Eq. (7) is studied, the optimal solution may consequently not be found. Both problems will be solved by a change of coordinates, see below.

In a control room, Eq. (7) is of no use since the coordinates of individual particles cannot be determined, and all information is instead obtained from the emittance measurement station. The equation will, however, be of great importance for the design of tuning bumps.

\section{A. Emittance function and coordinate normalization}

Given the macroparticle representation of the beam, the projected normalized emittance in the vertical plane at the end of the machine can be written 


$$
\begin{aligned}
\epsilon_{N}= & \gamma_{r}\left[\left(\sum_{k} w_{k}\left(y_{k}-\bar{y}\right)^{2}+\bar{\sigma}_{y y}\right)\left(\sum_{k} w_{k}\left(y_{k}^{\prime}-\bar{y}^{\prime}\right)^{2}+\bar{\sigma}_{y^{\prime} y^{\prime}}\right)\right. \\
& \left.-\left(\sum_{k} w_{k}\left(y_{k}-\bar{y}\right)\left(y_{k}^{\prime}-\bar{y}^{\prime}\right)+\bar{\sigma}_{y y^{\prime}}\right)^{2}\right]^{1 / 2}
\end{aligned}
$$

where $\gamma_{r}$ is the Lorentz factor, and $\bar{y}$ and $\bar{y}^{\prime}$ are the weighted average position and angle, respectively. For simplicity it has been assumed that the macroparticle weights are normalized such that their total weight is one. From the emittance definition it is clear that a "uniform" change of positions and/or angles does not affect the emittance. In other words, only the distribution of the macroparticles around the average in phase space $\left(y y^{\prime}\right)$ matters for the emittance. The $\bar{\sigma}$ 's are the weighted averages of the second momenta for all macroparticles. For an ideal beam their values are

$$
\bar{\sigma}_{y y}=\beta \frac{\epsilon_{N 0}}{\gamma_{r}}, \quad \bar{\sigma}_{y y^{\prime}}=\alpha \frac{\epsilon_{N 0}}{\gamma_{r}}, \quad \bar{\sigma}_{y^{\prime} y^{\prime}}=\gamma \frac{\epsilon_{N 0}}{\gamma_{r}},
$$

where the Twiss parameters $\alpha, \beta$, and $\gamma$ are determined by the optics of the machine. The minimum emittance

$$
\epsilon_{N 0}=\gamma_{r} \sqrt{\bar{\sigma}_{y y} \bar{\sigma}_{y^{\prime} y^{\prime}}-\bar{\sigma}_{y y^{\prime}}^{2}}
$$

is, as already mentioned, achieved whenever $y_{k}=A, y_{k}^{\prime}=$ $B$ for all $k$ with $A$ and $B$ being arbitrary constants.

In order to facilitate the design of emittance tuning bumps, the average position $\bar{y}$ and average angle $\bar{y}^{\prime}$ are subtracted from $y_{k}$ and $y_{k}^{\prime}$, respectively. In this way, only the deviation of positions and angles from the averages will be considered and a single optimum $\left(y_{k}=0, y_{k}^{\prime}=0\right)$ will exist. By Taylor expanding Eq. (8) around this optimum and dropping all terms of order three and higher, a secondorder approximation of the emittance is obtained:

$$
\begin{aligned}
\boldsymbol{\epsilon}_{N} \approx & \boldsymbol{\epsilon}_{N 0}+\gamma_{r}^{2} \frac{\bar{\sigma}_{y^{\prime} y^{\prime}}}{2 \epsilon_{N 0}} \sum_{k, k^{\prime}}\left(\delta_{k k^{\prime}}-w_{k^{\prime}}\right) w_{k} y_{k} y_{k^{\prime}} \\
& -\gamma_{r}^{2} \frac{\bar{\sigma}_{y y^{\prime}}}{\epsilon_{N 0}} \sum_{k, k^{\prime}}\left(\delta_{k k^{\prime}}-w_{k^{\prime}}\right) w_{k} y_{k} y_{k^{\prime}}^{\prime} \\
& +\gamma_{r}^{2} \frac{\bar{\sigma}_{y y}}{2 \epsilon_{N 0}} \sum_{k, k^{\prime}}\left(\delta_{k k^{\prime}}-w_{k^{\prime}}\right) w_{k} y_{k}^{\prime} y_{k^{\prime}}^{\prime} .
\end{aligned}
$$

Using the vector notation introduced above, this can be written on matrix form as

$$
\Delta \epsilon_{N}=\epsilon_{N}-\epsilon_{N 0} \approx \frac{1}{2} \tilde{\mathbf{y}}^{T} \mathbf{H} \tilde{\mathbf{y}}
$$

which is a good approximation of the emittance growth $\Delta \epsilon_{N}$ for small $\tilde{\mathbf{y}}$. The Hessian matrix $\mathbf{H}$ contains the second-order partial derivatives of the emittance function $\epsilon_{N}(\tilde{\mathbf{y}})$ and is explicitly

$$
\mathbf{H}=\gamma_{r}\left(\begin{array}{cc}
\gamma \mathbf{H}^{\prime} & -\alpha \mathbf{H}^{\prime} \\
-\alpha \mathbf{H}^{\prime} & \beta \mathbf{H}^{\prime}
\end{array}\right)
$$

where the matrix $\mathbf{H}^{\prime}$ is

$$
\begin{aligned}
\mathbf{H}^{\prime}= & \left(\begin{array}{cccc}
w_{1} & 0 & \ldots & 0 \\
0 & w_{2} & \ldots & 0 \\
\vdots & \vdots & \ddots & \vdots \\
0 & 0 & \ldots & w_{p}
\end{array}\right) \\
& -\left(\begin{array}{cccc}
w_{1}^{2} & w_{1} w_{2} & \ldots & w_{1} w_{p} \\
w_{2} w_{1} & w_{2}^{2} & \ldots & w_{2} w_{p} \\
\vdots & \vdots & \ddots & \vdots \\
w_{p} w_{1} & w_{p} w_{2} & \ldots & w_{p}^{2}
\end{array}\right)
\end{aligned}
$$

for a beam represented by $p$ macroparticles.

Observe that $\mathbf{H}^{\prime}$ and consequently $\mathbf{H}$ are both symmetric matrices. Since the Hessian $\mathbf{H}$ is calculated at a minimum point, it must be positive semidefinite; if not, there would be a direction along which further minimization could be achieved. As shown in [20], a symmetric positive semidefinite matrix has real non-negative eigenvalues and can be Schur decomposed:

$$
\mathbf{H}=\mathbf{Q D Q}^{T} .
$$

Here $\mathbf{Q}$ is an orthogonal matrix containing the eigenvectors of $\mathbf{H}$, while $\mathbf{D}$ is a diagonal matrix with the eigenvalues of $\mathbf{H}$ in increasing order in the diagonal. To further simplify the emittance growth expression of Eq. (12), a coordinate transformation, which normalizes the coordinates with respect to their effect on emittance growth, can be performed:

$$
\begin{aligned}
\Delta \boldsymbol{\epsilon}_{N} & \approx \frac{1}{2} \tilde{\mathbf{y}}^{T} \mathbf{Q D} \mathbf{Q}^{T} \tilde{\mathbf{y}}=\left(\frac{1}{\sqrt{2}} \mathbf{D}^{1 / 2} \mathbf{Q}^{T} \tilde{\mathbf{y}}\right)^{T}\left(\frac{1}{\sqrt{2}} \mathbf{D}^{1 / 2} \mathbf{Q}^{T} \tilde{\mathbf{y}}\right) \\
& =(\mathbf{M} \tilde{\mathbf{y}})^{T} \mathbf{M} \tilde{\mathbf{y}}=\mathbf{y}^{T} \mathbf{y}=|\mathbf{y}|^{2} .
\end{aligned}
$$

In this equation, $\mathbf{D}^{1 / 2}$ is the diagonal matrix whose diagonal elements are the square roots of the eigenvalues of $\mathbf{H}$. In this way

$$
\mathbf{M}=\frac{1}{\sqrt{2}} \mathbf{D}^{1 / 2} \mathbf{Q}^{T}
$$

defines the desired coordinate transformation. It should be pointed out that this transformation does not have an inverse. The reason is that the two first eigenvalues of $\mathbf{H}$ are zero and consequently the top two rows of $\mathbf{M}$ contain only zeros. From Eqs. (8) and (12), it is clear that the two eigenvectors of $\mathbf{H}$ with eigenvalue zero are related to the 2 degrees of freedom of uniform changes of positions and/or angles. If the Hessian matrix is explicitly determined, these two eigenvectors indeed turn out to be two "step functions," see Fig. 8. Along these two "dummy directions" emittance is constant.

When $\mathbf{M}$ has been calculated, it can be used to transform all seed and knob vectors to normalized space:

$$
\mathbf{S}=\mathbf{M} \tilde{\mathbf{S}}
$$




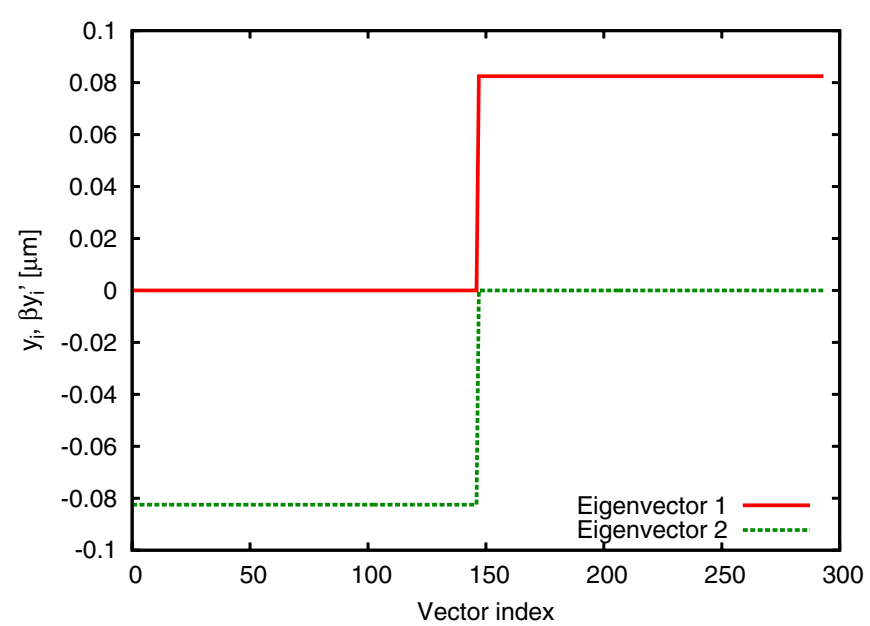

FIG. 8. The two eigenvectors of $\mathbf{H}$ with zero eigenvalues correspond to a uniform change of positions and angles, respectively.

$$
\mathbf{K}=\mathbf{M} \tilde{\mathbf{K}} .
$$

This coordinate normalization has a few very useful consequences related to the fact that $\Delta \epsilon_{N} \approx \mathbf{y}^{T} \mathbf{y}=|\mathbf{y}|^{2}$, and all calculations are from this point onwards carried out in the normalized coordinate space. One important consequence is that only one single point $(\mathbf{y}=0)$ corresponds to minimized emittance. Another effect of the normalization is that minimization of the norm of

$$
\mathbf{y}=\mathbf{s}_{i}+\mathbf{K} \mathbf{x}_{i}
$$

automatically minimizes emittance (to second order). These facts will be exploited in Sec. III C.

\section{B. Conjugate directions}

A third advantage of the normalized space becomes apparent when the important concept of conjugate directions [20,21] is introduced. As described in Sec. II B, a potential problem of the emittance tuning bumps is the convergence speed. Two knobs are noninterfering if the corresponding knob vectors are conjugate. Two vectors a and $\mathbf{b}$ are $\mathbf{H}$-conjugate if

$$
\mathbf{a}^{T} \mathbf{H b}=0 .
$$

If a general second-order function

$$
f(\mathbf{y})=f(0)+\left.\nabla f\right|_{0} \mathbf{y}+\left.\frac{1}{2} \mathbf{y}^{T} \mathbf{H}\right|_{0} \mathbf{y}
$$

is minimized by a line search along one of the directions a or $\mathbf{b}$ followed by a line search along the other direction, it will not be necessary to redo the minimization along the first direction if $\mathbf{a}$ and $\mathbf{b}$ are $\mathbf{H}$-conjugate [21]. In the normalized space, the Hessian of the emittance growth function $\Delta \epsilon_{N} \approx \mathbf{y}^{T} \mathbf{y}$ is simply $2 \mathbf{I}$ and two directions are conjugate if

$$
\mathbf{a}^{T} \mathbf{I} \mathbf{b}=\mathbf{a}^{T} \mathbf{b}=0,
$$

i.e. conjugate directions are orthogonal and vice versa. The equivalence of orthogonality and conjugacy is a major advantage of the normalized coordinates compared to the non-normalized ones. As will be seen below, it greatly simplifies the design of noninterfering knobs.

\section{SVD and principal directions}

The great advantages of the normalized coordinate space become clearly visible when singular value decomposition (SVD) [21] is used to decompose the seed matrix $\mathbf{S}$ and knob matrix K. The SVD algorithm decomposes an arbitrary matrix $\mathbf{A}$ into the product

$$
\mathbf{A}=\mathbf{U W} \mathbf{V}^{T} .
$$

Here $\mathbf{U}$ is a matrix whose orthogonal columns span the range of $\mathbf{A}$. The diagonal matrix $\mathbf{W}$ contains the real nonnegative singular values $\sigma_{i}$ of $\mathbf{A}$ in a decreasing order in the diagonal. $\mathbf{V}^{T}$ is a square orthogonal, hence normpreserving, matrix. If necessary, the SVD algorithm of, for example, OCTAVE [22] extends $\mathbf{U}$ such that a complete orthonormal basis is created. The corresponding rows added to $\mathbf{W}$ contain zeros only, thereby leaving the product UW unchanged.

Observe that the columns of $\mathbf{U}$ are eigenvectors of $\mathbf{A} \mathbf{A}^{T}$ :

$$
\begin{aligned}
\mathbf{A A}^{T} \mathbf{U} & =\mathbf{U W} \mathbf{V}^{T}\left(\mathbf{U W} \mathbf{V}^{T}\right)^{T} \mathbf{U}=\mathbf{U W} \mathbf{V}^{T} \mathbf{V} \mathbf{W}^{T} \mathbf{U}^{T} \mathbf{U} \\
& =\mathbf{U W W} \mathbf{W}^{T},
\end{aligned}
$$

where the third equality was obtained using the orthogonality of $\mathbf{U}$ and $\mathbf{V}$. WW $\mathbf{W}^{T}$ is a square diagonal matrix with the eigenvalues of $\mathbf{A} \mathbf{A}^{T}$ in the diagonal. Similarly, the columns of $\mathbf{V}$ are the eigenvectors of $\mathbf{A}^{T} \mathbf{A}$.

If the seed matrix $\mathbf{S}$ for a set of 100 random CLIC machines is decomposed with the SVD algorithm,

$$
\mathbf{S}=\mathbf{U}_{S} \mathbf{W}_{S} \mathbf{V}_{S}^{T},
$$

and its singular values are plotted, it becomes evident that they decrease very rapidly, see Fig. 9. The columns of $\mathbf{U}_{S}$ that are in the range of $\mathbf{S}$ (consequently having nonzero singular values) will from now on be referred to as the principal seed directions. The singular values can be interpreted as the importance of the corresponding columns of $\mathbf{U}$ with the first columns (with the highest singular values) being the most important. According to Eq. (24) the matrix $\mathbf{V}^{T}$ specifies the columns of $\mathbf{A}$ as linear combinations of the columns of $\mathbf{U W}$. A column of $\mathbf{U}$ multiplied by a relatively low singular value will have little significance for the linear combination compared to a column multiplied by a large singular value. By truncating $\mathbf{W}$, i.e., by replacing negligible singular values by zero, the number of degrees of freedom can be reduced without modifying the original problem considerably. An approximation of matrix $\mathbf{A}$ is thus given by 


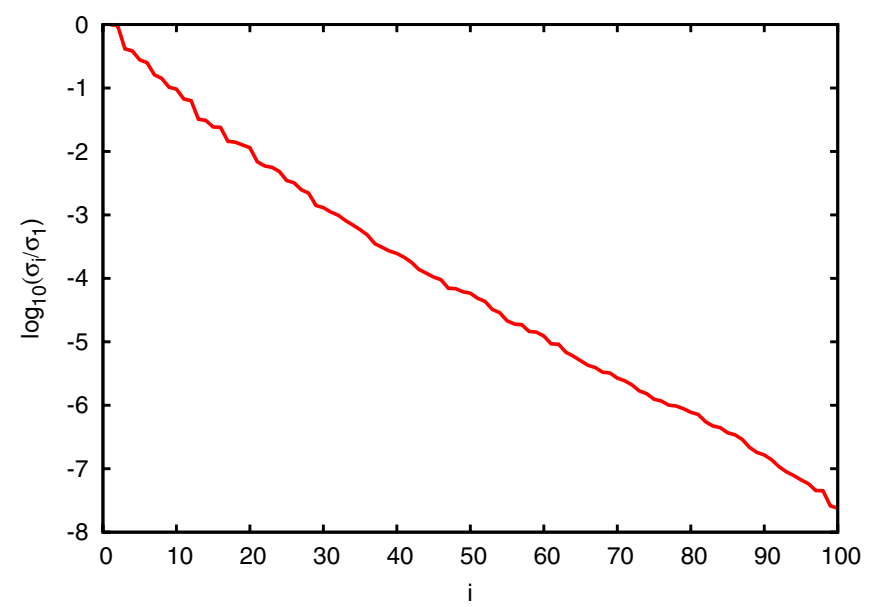

FIG. 9. Relative singular values $\left(\sigma_{i} / \sigma_{1}\right)$ of the seed matrix corresponding to 100 CLIC machines obtained after beam-based alignment of randomly misaligned machines. The importance of the majority of directions is negligible.

$$
\mathbf{A}^{\prime}=\mathbf{U W}^{\prime} \mathbf{V}^{T},
$$

where $\mathbf{W}^{\prime}$ is the matrix $\mathbf{W}$ with elements $\sigma_{i}$ such that $\sigma_{i}<$ $\delta \sigma_{1}$, for some small $\delta$, replaced by zero.

The rapid decrease in magnitude of the singular values indicates that the number of degrees of freedom is very limited even for a large set of random seeds. This suggests that it is sufficient to minimize emittance along a few important principal seed directions to reduce the average emittance growth of this particular set of seeds to an acceptable level. To quantify this statement, the seed vectors are transformed to the $\mathbf{U}_{S}$-basis:

$$
\mathbf{S}^{\prime}=\mathbf{U}_{S}^{T} \mathbf{S}
$$

The transformation obviously preserves the norm of each seed vector, and consequently emittance growth still equals $\left|\mathbf{s}^{\prime}\right|^{2}$ to second order. For this particular set of seeds, the importance of an arbitrary direction $\hat{\mathbf{a}}$ can be determined by studying how well the average emittance growth of all seeds can be minimized along it. By minimizing the norm of

$$
\mathbf{y}=\mathbf{s}_{i}^{\prime}+\hat{\mathbf{a}} x_{i}
$$

for seed $i$, the unique emittance growth minimum is found. The least-square solution for each seed is given by

$$
x_{i}=-\left(\hat{\mathbf{a}}^{T} \hat{\mathbf{a}}\right)^{-1} \hat{\mathbf{a}}^{T} \mathbf{s}_{i}^{\prime}=-\hat{\mathbf{a}}^{T} \mathbf{s}_{i}^{\prime},
$$

where it has been assumed that $\hat{\mathbf{a}}$ is of unit length. The set of seed vectors obtained after correction along direction $\hat{\mathbf{a}}$ is in matrix form

$$
\mathbf{S}^{\prime}-\hat{\mathbf{a}} \hat{\mathbf{a}}^{T} \mathbf{S}^{\prime}=\left(1-\hat{\mathbf{a}} \hat{\mathbf{a}}^{T}\right) \mathbf{S}^{\prime}=\left(1-\hat{\mathbf{a}} \hat{\mathbf{a}}^{T}\right) \mathbf{W}_{S} \mathbf{V}_{S}^{T},
$$

where Eqs. (26) and (28) have been used. The squared norm of column $i$ of this matrix is the emittance growth of seed $i$ after correction. Consequently, the average emittance growth of $N$ corrected seeds is

$$
\begin{aligned}
\overline{\Delta \epsilon_{N}} & \approx \frac{1}{N} \operatorname{Tr}\left\{\left[\left(1-\hat{\mathbf{a}} \hat{\mathbf{a}}^{T}\right) \mathbf{W}_{S} \mathbf{V}_{S}^{T}\right]^{T}\left[\left(1-\hat{\mathbf{a}} \hat{\mathbf{a}}^{T}\right) \mathbf{W}_{S} \mathbf{V}_{S}^{T}\right]\right\} \\
& =\frac{1}{N}\left[\operatorname{Tr}\left(\mathbf{W}_{S}^{T} \mathbf{W}_{S}\right)-\operatorname{Tr}\left(\mathbf{W}_{S}^{T} \hat{\mathbf{a}} \hat{\mathbf{a}}^{T} \mathbf{W}_{S}\right)\right] \\
& =\frac{1}{N} \sum_{i}\left(1-a_{i}^{2}\right) \sigma_{i}^{2},
\end{aligned}
$$

where $a_{i}$ are the components of $\hat{\mathbf{a}}$ in the $\mathbf{U}_{S}$-basis. The second equality of Eq. (32) was obtained by using the orthogonality of $\mathbf{V}_{S}$, the unit norm of $\hat{\mathbf{a}}$, and the fact that $\operatorname{Tr}\left(\mathbf{A} \mathbf{A}^{T}\right)=\operatorname{Tr}\left(\mathbf{A}^{T} \mathbf{A}\right)$ for any matrix $\mathbf{A}$. Under the assumption that the singular values $\sigma_{i}$ are sorted in decreasing order, average emittance growth is obviously minimized for $a_{1}=1$ and $a_{i}=0, i>1$. The optimal choice of $\hat{\mathbf{a}}$ is therefore in the direction of the first column $\mathbf{u}_{1}$ of $\mathbf{U}$, i.e., along the most important principal seed direction. In a similar fashion, it can be argued that, when minimization along $\mathbf{u}_{1}$ has been performed, the optimal direction is $\mathbf{u}_{2}$ and so on. By optimizing along the $n$ most important directions, the average emittance growth can be reduced to

$$
\overline{\Delta \epsilon_{N}} \approx \frac{1}{N} \sum_{i=n+1}^{N} \sigma_{i}^{2}
$$

The minimum achievable emittance growth as a function of $n$ is plotted for both CLIC and ILC in Fig. 10. This plot shows that, by using the most important principal directions, as few as four directions is sufficient to achieve an emittance growth of less than $0.04 \mathrm{~nm}$ for ILC. For CLIC, optimization along ten directions is sufficient for reducing emittance growth to less than $0.16 \mathrm{~nm}$. For such low emittances, effects of noise in the emittance measurement and other dynamic imperfections will be the dominating

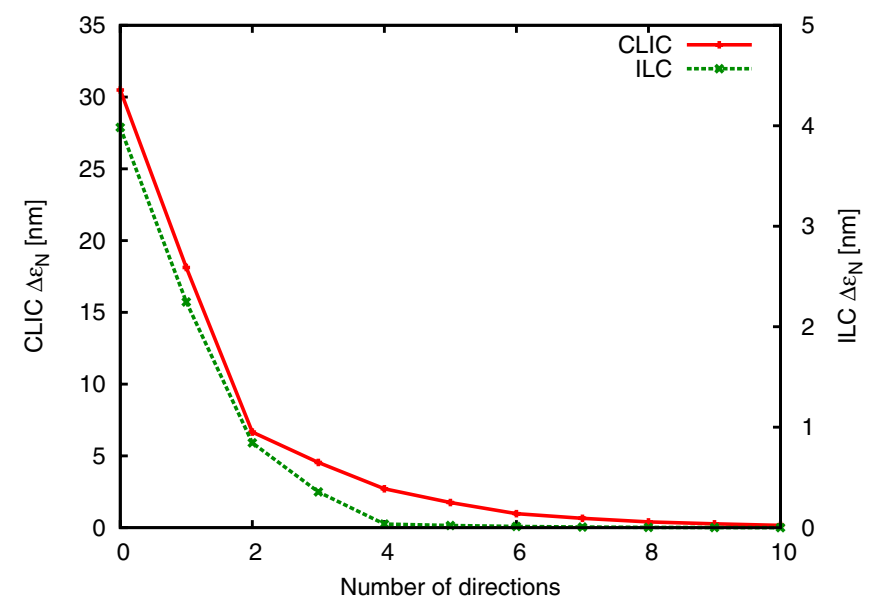

FIG. 10. Second-order approximation of the remaining emittance growth after optimization along the $n$ most important principal seed directions. Observe that the emittance scales for CLIC and ILC differ. Optimization along ten directions is for CLIC enough to reduce emittance growth to $0.16 \mathrm{~nm}$. For ILC four directions are sufficient to reduce emittance growth to less than $0.04 \mathrm{~nm}$. 
source of emittance growth, and at a certain point it is meaningless to try to achieve an even lower theoretical minimum by optimizing along additional directions.

At first sight, it might seem that the principal directions would be strongly dependent on the particular set of seeds being studied. To show that this is not the case and indicate that the principal directions of a large set of seeds are rather a property of the ideal machine optics and the beam-based alignment scheme used, two different studies were carried out.

The first study was carried out to determine how many principal seed directions are useful for emittance reduction as a function of the number of seeds. The minimum number of directions required to reduce emittance growth to less than $0.2 \mathrm{~nm}$ was therefore determined, and as shown in Fig. 11 this number converges fast as the number of seeds increase, both for CLIC and ILC. If an infinite number of seeds can be corrected to an acceptable average emittance along a small number of principal directions, these directions must be a property of the machine optics and the initial alignment procedures. Consequently, they could be obtained by simulations prior to machine start-up. Another way of showing this is to divide 100 ILC seeds into two groups $\mathbf{S}_{1}$ and $\mathbf{S}_{2}$ of 50 vectors each. When the principal seed directions of these two groups are calculated separately, clear similarities are found. More importantly, if the first group of seeds is corrected along either of the sets of directions, very similar performance is obtained, see Fig. 12. If these directions can be constructed as linear combinations of a given set of knob vectors $\mathbf{K}$, a new set of optimal knobs can be created. Observe that, since normalized coordinates are used, the principal directions are not only orthogonal but also conjugate cf. Sec. III B. Consequently, the new knobs will be nearly noninterfering

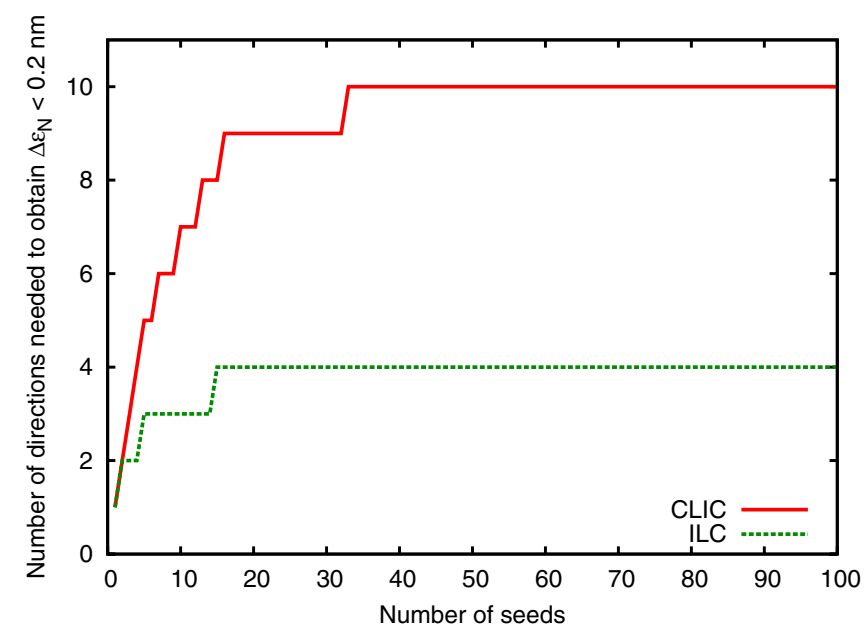

FIG. 11. The minimum number of principal seed directions required to reduce emittance growth to less than $0.2 \mathrm{~nm}$ quickly converges, both for CLIC and ILC. Even for a large number of seeds, four directions are sufficient for ILC. For CLIC the corresponding number is ten.

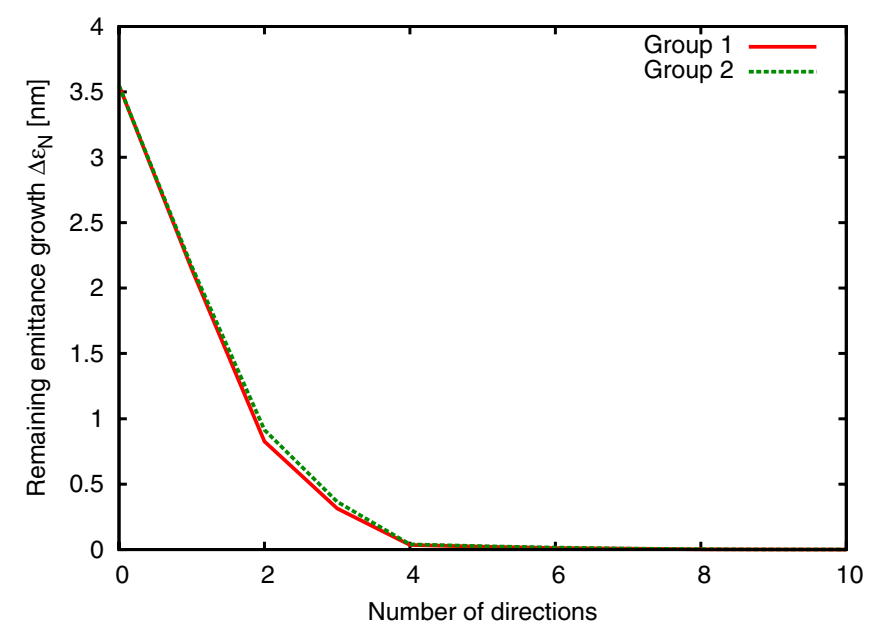

FIG. 12. Emittance reduction of 50 ILC seeds along the principal directions of the group of seeds itself (group 1) compared to along the principal directions obtained from a separate set of seeds (group 2).

in the regime where the second-order emittance approximation is accurate, and the convergence will be almost instant.

Just as for the seed matrix, SVD of the knob matrix is very useful for the design of emittance bumps. The decomposition gives

$$
\mathbf{K}=\mathbf{U}_{K} \mathbf{W}_{K} \mathbf{V}_{K}^{T},
$$

where the order of the columns of $\mathbf{U}_{K}$ is such that the first columns correspond to the directions that can most easily be generated with the given set of knobs. In case of knobs controlling element displacements, this means that the first column is the one which is produced by the smallest rms element displacement. This is seen by noting that a unit step along each direction is achieved by a linear combination

$$
\mathbf{U}_{K}=\mathbf{K} \mathbf{V}_{K} \mathbf{W}_{K}^{-1}
$$

of the given knobs. The required rms knob adjustment for a certain direction is thus seen to be the square root of the corresponding diagonal element of the matrix

$$
\left(\mathbf{V}_{K} \mathbf{W}_{K}^{-1}\right)^{T} \mathbf{V}_{K} \mathbf{W}_{K}^{-1}=\left(\mathbf{W}_{K}^{-1}\right)^{T} \mathbf{W}_{K}^{-1} .
$$

Consequently, the inverse singular value gives a measure of how much a knob has to be adjusted to generate a unit step along the corresponding column of $\mathbf{U}_{K}$. For an aligned machine such a unit step corresponds to a unit change of emittance, and the first columns therefore produce maximum emittance change per unit of knob adjustment. The directions with the highest singular values will from now on be referred to as the strongest directions. Below will be shown that the strongest directions are not always useful for emittance reduction.

In analogy with the notation above, the columns of $\mathbf{U}_{K}$ that are in the range of $\mathbf{K}$ specify what will be referred to as 
principal knob directions. All principal knob directions have nonzero singular values.

\section{Construction of optimal knobs}

As mentioned earlier, $\mathbf{U}_{K}$ forms a complete orthonormal basis. Transformation of the seed vectors to the $\mathbf{U}_{K}$-basis gives

$$
\mathbf{S}^{\prime}=\mathbf{U}_{K}^{T} \mathbf{S}
$$

It can be seen that the largest possible average emittance reduction along $\mathbf{u}_{i}$ (column $i$ of $\mathbf{U}_{K}$ ) is equal to the squared norm of row $i$ of $\mathbf{S}^{\prime}$ divided by the total number of seeds. In this way the achievable emittance reduction along each column of $\mathbf{U}_{K}$ may be calculated for the two previously mentioned groups of ILC seeds, see Fig. 13. Observe that, if the singular value corresponding to $\mathbf{u}_{i}$ is zero, i.e., if $\mathbf{u}_{i}$ is not a principal knob direction, the given set of knobs cannot be used to correct emittance along it.

For a completely general set of knobs, there is no particular correlation between the strength of a principal direction and its usefulness for emittance reduction. In this case, however, the knobs are based on quadrupole displacements, and since undesired displacements of quadrupoles is also one of the main sources of emittance growth for the initial seeds, there is a visible correlation.

The two curves in Fig. 13 are very similar. In both cases, the same knob directions have to be used to achieve effective emittance reduction, once again showing the fundamentality of the principal seed directions. In order to reach as low emittances as achievable along the four most important principal seed directions (cf. Fig. 10), the ten strongest principal knob directions have to be used.

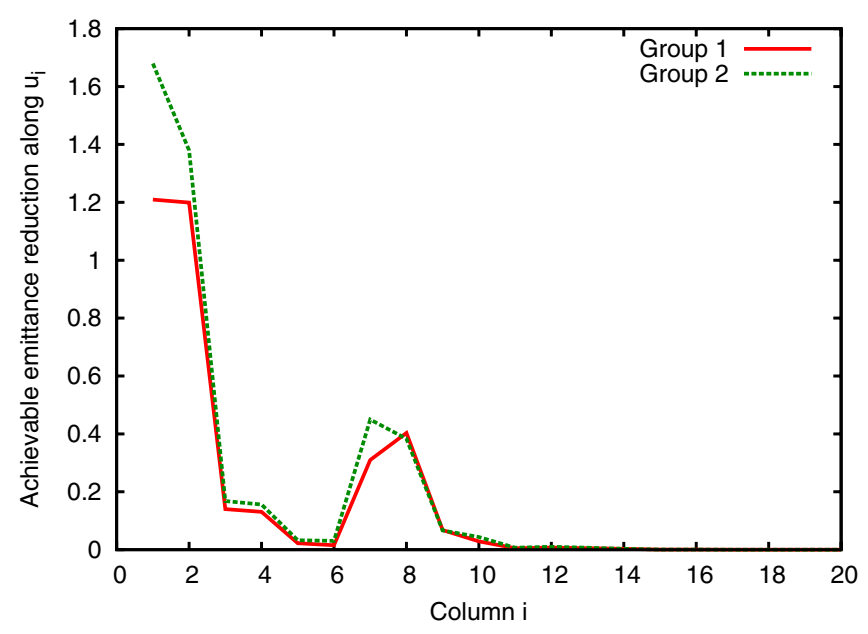

FIG. 13. Maximum achievable emittance reduction along the columns of $\mathbf{U}_{K}$ for two separate groups of ILC seeds. Note how the strong directions 5 and 6 are nearly useless for emittance reduction. The reason is that the seeds that need to be corrected have very small components along these directions. They could otherwise have been used to correct emittance without using large quadrupole displacements.
For CLIC and a set of knobs based on accelerating structure displacements, a plot like Fig. 13 shows an even stronger correlation between the strength of a knob direction and its usefulness for emittance reduction. This may indicate that most of the emittance growth in the CLIC main linac is caused by displacements of accelerating structures. The strongest principal knob directions are in this case almost as efficient as the principal seed directions. New knobs that optimize emittance along these knob directions are simply constructed as linear combinations of the old knobs according to Eq. (35). The number of principal knob directions to use is a balance between the desired emittance reduction and the acceptable element displacements.

As described above, the principal seed directions are the optimal directions for emittance reduction. To construct new knobs that optimize emittance along these most efficient directions, suitable linear combinations of the available knobs have to be determined. An exact construction is often not possible, in particular, if the knob range is limited. An approximate solution may, however, be obtained by solving

$$
\mathbf{U}_{S}=\mathbf{K N}
$$

for $\mathbf{N}$. By using the SVD of $\mathbf{K}$ [Eq. (34)], the equation may be solved in the least-square sense, giving the linear combinations that most closely resemble the desired principal seed directions. If all principal seed directions are in the range of $\mathbf{K}$, an exact solution can be found, and the optimal knobs can be constructed. The solution may, however, require very large knob adjustments. This problem may be avoided by truncating small values of $\mathbf{W}_{K}$, and the solution becomes

$$
\mathbf{N}=\mathbf{V}_{K}\left(\mathbf{W}_{K}^{\prime}\right)^{-1} \mathbf{U}_{K}^{T} \mathbf{U}_{S},
$$

where $\left(\mathbf{W}_{K}^{\prime}\right)^{-1}$ is obtained by inverting the nonzero elements of the truncated matrix $\mathbf{W}_{K}^{\prime}$. If $\mathbf{W}_{K}^{\prime}$ is not square, it must also be transposed.

By truncating more singular values, i.e. by excluding more of the weak principal knob directions, the required knob settings become smaller. At the same time, however, the components of the principal seed directions along the excluded principal knob directions can no longer be reproduced and the construction of the former becomes less precise. Each knob created using Eq. (39) will therefore only reproduce the projection of a principal seed direction on the subset of the strongest (not excluded) principal knob directions. These knobs may not be optimal in terms of emittance reduction capability. Furthermore, they may not be orthogonal which could lead to slower convergence. In order to obtain the optimal (in terms of emittance reduction performance) orthogonal set of knobs which can be constructed using only the strongest principal knob directions, it is necessary to use Eq. (32) once more. From this equation it is clear that the optimal unit length direction $\hat{\mathbf{a}}$, with components $a_{i}$ in the $\mathbf{U}_{S}$-basis, is found by max- 
imizing the total emittance reduction

$$
\sum_{i} a_{i}^{2} \sigma_{i}^{2}=\left(\mathbf{W}_{S}^{T} \mathbf{a}\right)^{T} \mathbf{W}_{S}^{T} \mathbf{a}
$$

where the matrix formulation is obtained by using that the $\sigma_{i}$ are the singular values of $\mathbf{S}$ (diagonal elements of $\mathbf{W}_{S}$ ). The direction â should, in this case, be limited to a linear combination of a subset $\mathbf{U}_{K}^{\prime}$ consisting of the strongest principal knob directions:

$$
\hat{\mathbf{a}}=\mathbf{U}_{K}^{\prime} \mathbf{z}
$$

with $|\mathbf{z}|=1$. By using that $\hat{\mathbf{a}}=\mathbf{U}_{S} \mathbf{a}$ and that $\mathbf{U}_{S}$ is orthonormal, it is possible to express $\mathbf{a}$ in terms of $\mathbf{z}$ :

$$
\mathbf{a}=\mathbf{U}_{S}^{T} \mathbf{U}_{K}^{\prime} \mathbf{z}
$$

The maximization problem may now be written

$$
\max _{|\mathbf{a}|=1}\left(\sum_{i} a_{i}^{2} \sigma_{i}^{2}\right)=\max _{|\mathbf{z}|=1}\left(\mathbf{z}^{T} \mathbf{\Psi} \mathbf{z}\right),
$$

where $\boldsymbol{\Psi}$ is obtained using Eqs. (40) and (42):

$$
\boldsymbol{\Psi}=\left(\mathbf{U}_{K}^{\prime}\right)^{T} \mathbf{U}_{S} \mathbf{W}_{S} \mathbf{W}_{S}^{T} \mathbf{U}_{S}^{T} \mathbf{U}_{K}^{\prime}=\left(\mathbf{U}_{K}^{\prime}\right)^{T} \mathbf{S} \mathbf{S}^{T} \mathbf{U}_{K}^{\prime} .
$$

According to [20], the maximum of a quadratic form as in the right-hand side of Eq. (43) is equal to the highest eigenvalue of the symmetric matrix $\boldsymbol{\Psi}$. The maximum is obtained for the corresponding eigenvector $\mathbf{z}=\mathbf{e}$, and the optimal knob that can be constructed as a linear combination of the principal knob directions in $\mathbf{U}_{K}^{\prime}$ is, consequently, $\hat{\mathbf{a}}=\mathbf{U}_{K}^{\prime} \mathbf{e}$. When optimization along this direction has been performed, the best remaining direction is obtained by using the second eigenvector (with second highest eigenvalue) of $\boldsymbol{\Psi}$. Since $\boldsymbol{\Psi}$ is symmetric, the eigenvectors can be obtained by SVD as the columns of $\mathbf{U}_{\Psi}$ and the eigenvalues, which in this case are equal to the singular values, as the diagonal elements of $\mathbf{W}_{\Psi}$ [20]. The eigenvalue of an eigenvector $\mathbf{e}$ can be interpreted as the total achievable emittance reduction for all seeds along the direction $\hat{\mathbf{a}}=$ $\mathbf{U}_{K}^{\prime} \mathbf{e}$. Using Eq. (35), the whole set of optimal directions can be written

$$
\mathbf{R}=\mathbf{U}_{K}^{\prime} \mathbf{U}_{\Psi}=\mathbf{K} \mathbf{V}_{K}\left(\mathbf{W}_{K}^{\prime}\right)^{-1} \mathbf{U}_{\Psi},
$$

where $\mathbf{W}_{K}^{\prime}$ are the rows of $\mathbf{W}_{K}$ corresponding to the columns (principal directions) of $\mathbf{U}_{K}^{\prime}$.

In analogy with the truncation of singular values mentioned above, the number of principal knob directions in $\mathbf{U}_{K}^{\prime}$ is chosen as a balance between emittance reduction performance and acceptable knob adjustments. Under the assumption that the constructed knobs converge fast, their emittance reduction performance may be estimated by the theoretically achievable reduction along the subset of principal directions. From the discussion above, the maximum total emittance reduction for all seeds is seen to be the sum of the eigenvalues of $\boldsymbol{\Psi}$. The ideal settings of the new optimal knobs for all seeds are obtained by finding the matrix $\mathbf{X}$ which minimizes the norms of the columns of

$$
\mathbf{S}+\mathbf{R X} \text {. }
$$

Since the columns of $\mathbf{R}$ are orthonormal, the least-square solution is

$$
\mathbf{X}=-\left(\mathbf{R}^{T} \mathbf{R}\right)^{-1} \mathbf{R}^{T} \mathbf{S}=-\mathbf{R}^{T} \mathbf{S},
$$

and the optimal settings of the original knobs $\mathbf{K}$ (corresponding to, e.g., accelerating structure displacements) are obtained from Eqs. (45) and (47) as

$$
\mathbf{V}_{K}\left(\mathbf{W}_{K}^{\prime}\right)^{-1} \mathbf{U}_{\Psi} \mathbf{X}=-\mathbf{V}_{K}\left(\mathbf{W}_{K}^{\prime}\right)^{-1} \mathbf{U}_{\Psi} \mathbf{R}^{T} \mathbf{S}
$$

Based on theoretical estimates of emittance reduction performance (sum of eigenvalues of $\boldsymbol{\Psi}$ ) and required knob adjustments [Eq. (48)], a reasonable number of principal knob directions may be chosen. Using these directions, an equal number of orthogonal knobs may be constructed as described above. Observe, however, that these knobs are not all necessarily efficient. While the first columns of $\mathbf{R}$ are efficient directions (high eigenvalues of $\boldsymbol{\Psi}$ ), the last columns may be nearly useless (low eigenvalues). As shown in Fig. 13, a strong principal knob direction is not always useful for emittance reduction and may constitute a nearly useless degree of freedom. In addition, the required corrections along different directions may be correlated such that nearly the same ratio appears between these corrections for all seeds. Consequently, one knob may be used to correct along several directions simultaneously, independent of the seed. The number of efficient knobs will therefore be smaller than the number of strong principal knob directions. The most efficient knobs are automatically identified by the method described above.

To construct optimal knobs, it is apparently first necessary to choose a reasonable number of strong principal knob directions. Using these, an equal number of knobs can then be constructed. As explained above, some of these knobs may not be very efficient, and in order to determine how many to use, their individual emittance reduction performance, determined by the corresponding eigenvalues of $\boldsymbol{\Psi}$, can be studied. As will be seen in the next section, the number of principal knob directions and the number of useful knobs are not equal for ILC knobs based on quadrupole displacements. This was expected since Fig. 12 showed that as few as four principal directions are sufficient for emittance reduction, while Fig. 13 showed that the ten strongest principal knob directions have to be used. For CLIC, all knobs (based on structure displacements) created as linear combinations of the strongest principal knob directions are useful. Instead of simply testing what the optimal numbers are, some more elaborate ways of finding the most efficient knobs were also studied. These used a penalty function based on the strength of each principal knob direction instead of completely removing all but the strongest directions. This method could only improve knobs marginally. 


\section{APPLICATIONS TO FUTURE LINEAR COLLIDERS}

The usefulness of the above described method can be shown by a few applications to CLIC and ILC. As initially described, emittance tuning bumps will be very important for CLIC and may also be useful for ILC. Because of the differences between the machines, e.g., in terms of constraints on how elements can be moved, the optimal knob design is completely different in the two cases. Under the assumption that emittance measurements are exact, the construction of very efficient knobs is however possible for both machines.

\section{A. CLIC}

\section{Orthogonalization of emittance tuning bumps}

As a first example of how to design knobs for CLIC with the method described above, the use of ten equidistant accelerating structures is investigated. In this context equidistant refers to the number of betatron oscillations (the number of quadrupoles). The most straightforward way to use the structures is to control the vertical displacement of each of them with a knob. For the simulations described here, it was assumed that each knob adjustment was followed by one-to-one steering in order to resteer the beam to the BPM centers. In reality, a faster method will have to be used to keep the beam close to the reference trajectory. Already this trivial use of the structures is very efficient for reducing emittance. The convergence speed and final emittance can, however, be significantly improved by constructing new orthogonal knobs, see Fig. 14. The construction of optimal knobs was carried out using the method of Sec. III, i.e., by SVD of the normalized knob matrix, followed by calculation of $\boldsymbol{\Psi}$ [cf. Eq. (44)] and its SVD. All ten principal knob directions were used, as were all ten constructed orthogonal knobs. In this case, the same 100 seeds were used for determination of principal seed directions as the ones that were then corrected. By dividing the set of seeds into two groups, as was done for ILC in Sec. III C, it was seen that the first group of seeds could be corrected equally well using principal seed directions from either of the groups.

In Fig. 14 a concave edge can be seen just between the first and the second iteration (after ten optimization steps). This indicates that convergence could become slightly faster if a few less knobs were used. Fewer knobs would, however, lead to a slightly higher theoretical minimum emittance. In order to improve convergence speed and keep emittance performance unchanged, the order of the knobs could instead be modified. This kind of fine-tuning of the knobs is not analyzed further since statistical fluctuations (in a study of 100 seeds) may affect the outcome. Furthermore, a small increase in emittance may be negligible compared to additional emittance growth caused by imperfections such as noise in the emittance measurement.

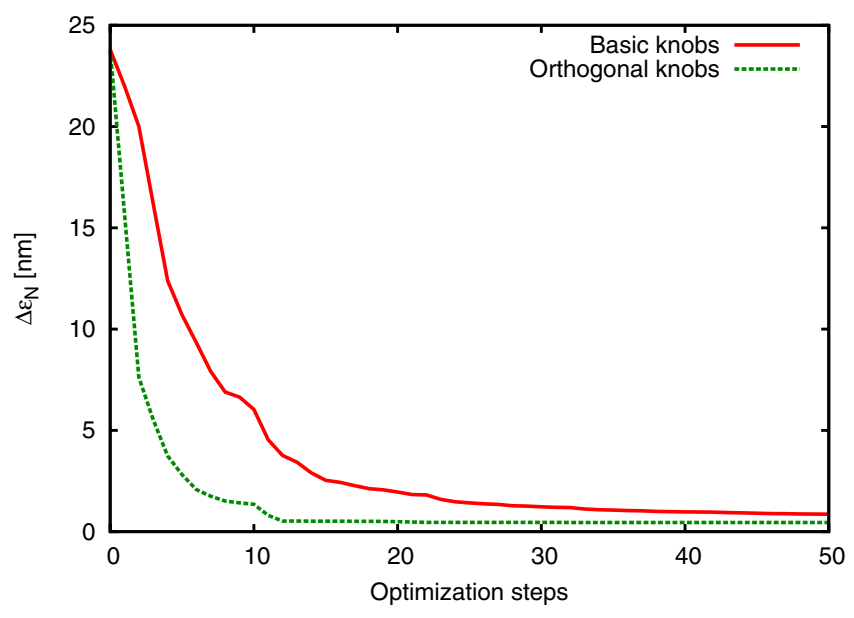

FIG. 14. Basic use of ten structures each controlled by a knob compared to using orthogonalized knobs each controlling all ten structures. In one iteration, the basic method reduces emittance growth to $6.0 \mathrm{~nm}$, while orthogonal knobs reduce growth to $1.3 \mathrm{~nm}$. With the new knobs, emittance growth converges rapidly to $0.44 \mathrm{~nm}$, in perfect agreement with theoretical predictions obtained from the eigenvalues of $\boldsymbol{\Psi}$. The convergence for the basic knobs is clearly slower with a final emittance growth of $0.87 \mathrm{~nm}$.

It is important to notice that, even though convergence speed and emittance reduction is very good, these knobs will not work because of the large accelerating structure displacements they require. In Fig. 5 the rms displacements for each seed using these particular knobs were shown. The displacements exceed what is acceptable by 2 orders of magnitude. For the basic knobs, the displacements are approximately half of the displacements for the orthogonalized knobs.

\section{Reduction of accelerating structure displacements}

As described in Sec. III C, the required knob adjustments grow with the index of the principal knob directions. By limiting the number of such directions, it is therefore possible to reduce the large displacements mentioned above. With only ten accelerating structures, this will, however, not be sufficient to obtain acceptable displacements and emittance simultaneously. Another way to solve the problem is by letting each knob control the displacement of a group of structures instead of only one. All structures should be positioned at the same, or nearly the same, betatron phase such that they have an equivalent effect on the beam at the end of the linac. This will reduce the required displacements by a factor roughly equal to the number of structures per group, while emittance reduction performance will be nearly unaffected. Finding big groups of "equivalent" accelerating structures may, due to variations in betatron frequency within each bunch of the beam, be difficult. Instead any large set of accelerating structures is chosen, and the procedure described in the previous section is used to create optimal knobs. 


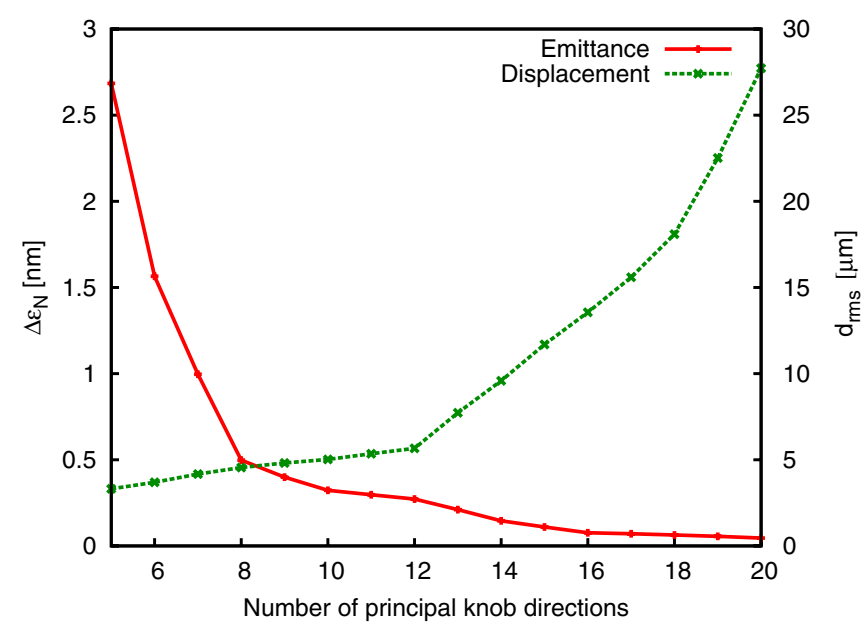

FIG. 15. Estimate of the average rms displacement and the average remaining emittance growth of 100 CLIC seeds for different numbers of principal knob directions.

In this case, all structures positioned next to (in the downstream direction) a focusing quadrupole were chosen. In total, this makes 662 accelerating structures. The normalized knob vectors were calculated, and SVD was carried out. The matrix $\boldsymbol{\Psi}$ was determined for different numbers of principal knob directions (numbers of nontruncated elements of $\mathbf{W}_{K}$ ), and the remaining emittance growth and structure displacements were calculated under the assumption that all constructed knobs were used, see Fig. 15. The plot shows that a reasonable number of knobs and principal knob directions is ten, giving an rms structure displacement of $5.0 \mu \mathrm{m}$ and a remaining emittance growth of $0.32 \mathrm{~nm}$. Independent variation of the number of principal knob directions and the number of constructed knobs

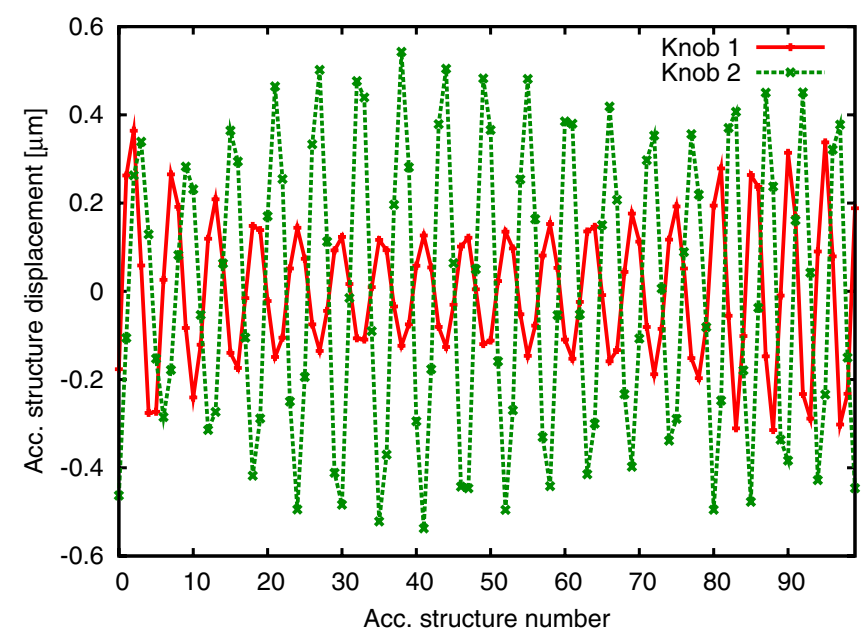

FIG. 16. Accelerating structure displacements of the 100 first structures (positioned next to the 100 first focusing quadrupoles) for the two most efficient knobs. The displacements are seen to vary approximately with the betatron frequency of the CLIC main linac. was tested without significant improvements. As an example, the rms displacement was estimated to $4.7 \mu \mathrm{m}$ and the emittance growth to $0.44 \mathrm{~nm}$ for the eight most efficient knobs constructed using the ten strongest principal knob directions.

The knob coefficients (accelerating structure displacements) obtained using ten principal knob directions and ten knobs seem to vary approximately with the betatron frequency of the linac but with an additional amplitude modulation, see Fig. 16. Intuitively, this appears to be an efficient way of assembling a large number of structure displacements into one knob, thus illustrating how the design strategy identifies equivalent structures and constructs efficient knobs.

Simulations confirmed the estimates of Fig. 15 and showed that the convergence is excellent. Apart from converging fast, the new knobs require acceptable structure displacements and reduce emittance growth to very low levels, see Figs. 17-19.

During the simulations described above, the beam was represented by 147 macroparticles. The initial distribution of these macroparticles was obtained by dividing the incoming beam into 21 slices and representing the particles of each slice by 7 macroparticles. In order to understand whether 147 macroparticles is enough to represent the beam and correctly construct optimal knobs, the knobs constructed using the 147-macroparticle representation were used to correct 100 seeds for a 341-macroparticle and a 969-macroparticle representation of the beam. The knobs were for both of these more accurate beam models shown to perform in the exact same way as for the 147macroparticle beam representation that was used to construct the knobs. The results shown in Fig. 17 were obtained by correction of a set of seeds along principal knob directions obtained from the set itself. The performance of

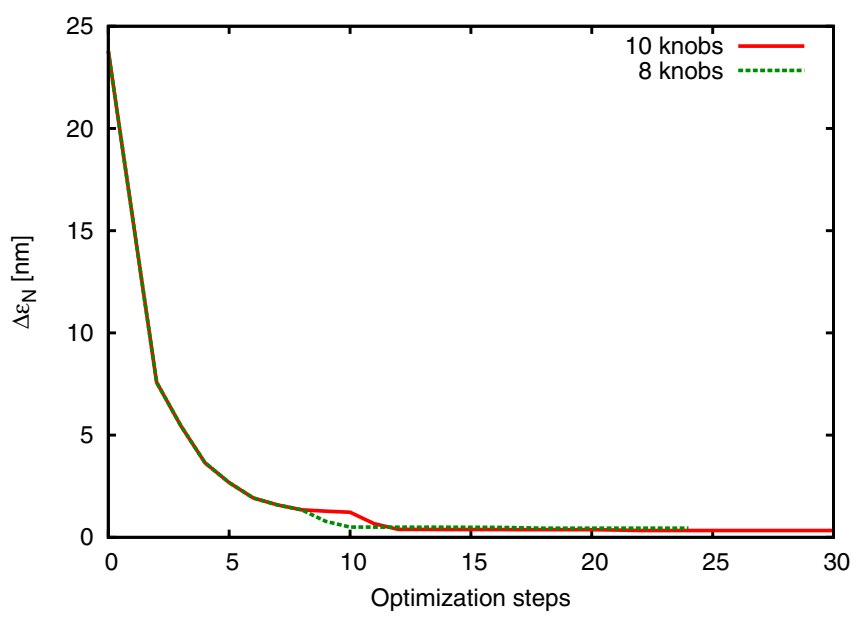

FIG. 17. Emittance growth reduction obtained using eight or ten knobs based on the ten strongest principal knob directions of a set of 662 knob vectors. For ten knobs the final emittance is lower, but convergence is slightly slower. 


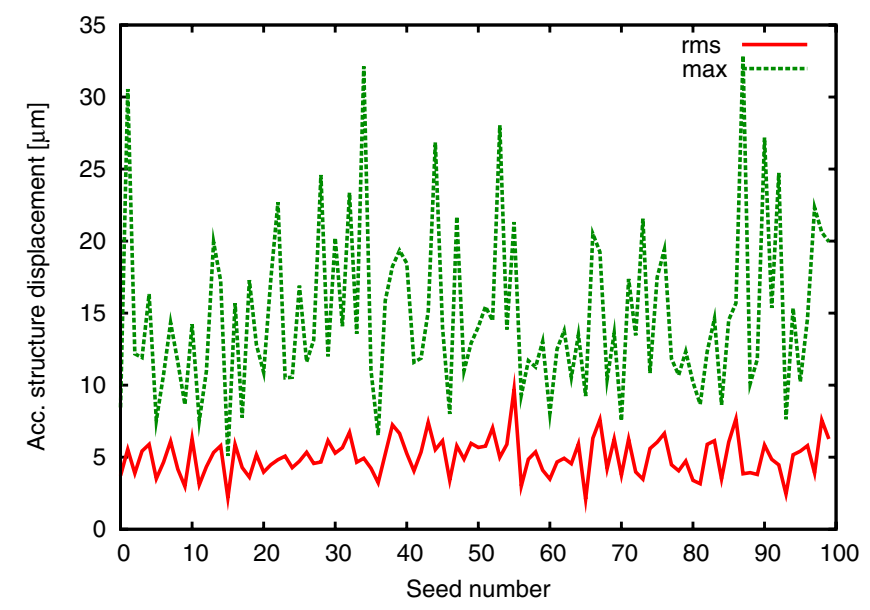

FIG. 18. Root-mean-square and maximum displacements of 662 accelerating structures controlled by ten knobs after correction of each of 100 seeds.

knobs constructed using a separate set of seeds was, however, seen to be nearly identical. Figure 20 shows that 50 seeds in the 969-macroparticle representation could be tuned either by optimal knobs obtained from the 50 seeds themselves or, with nearly the same performance, by knobs obtained from 50 other seeds in the 147-macroparticle representation.

Tests were also carried out to show that the tolerance to limitations in minimum mover step size is excellent. Ideally, the mover step size is infinitesimally small, giving an emittance growth of $0.335 \mathrm{~nm}$. For a step size of $1 \mu \mathrm{m}$, the emittance growth was $0.338 \mathrm{~nm}$. Step sizes of 2 and $4 \mu \mathrm{m}$ gave emittance growths of 0.344 and $0.371 \mathrm{~nm}$, respectively.

By using PLACET [19], a beam was tracked through the linac for each of the 100 seeds, taking into account the corrections obtained using the new emittance tuning

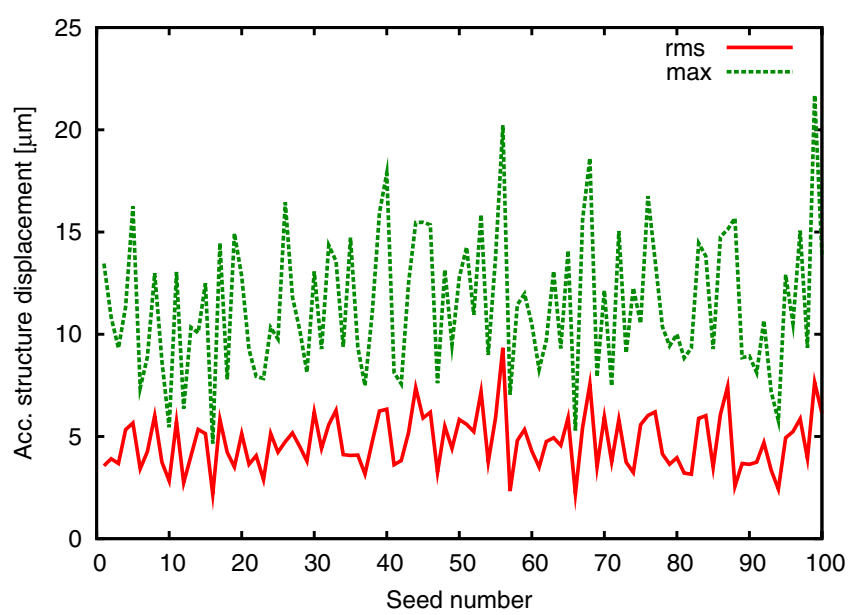

FIG. 19. Root-mean-square and maximum displacements of 662 accelerating structures controlled by eight knobs after correction of each of 100 seeds.

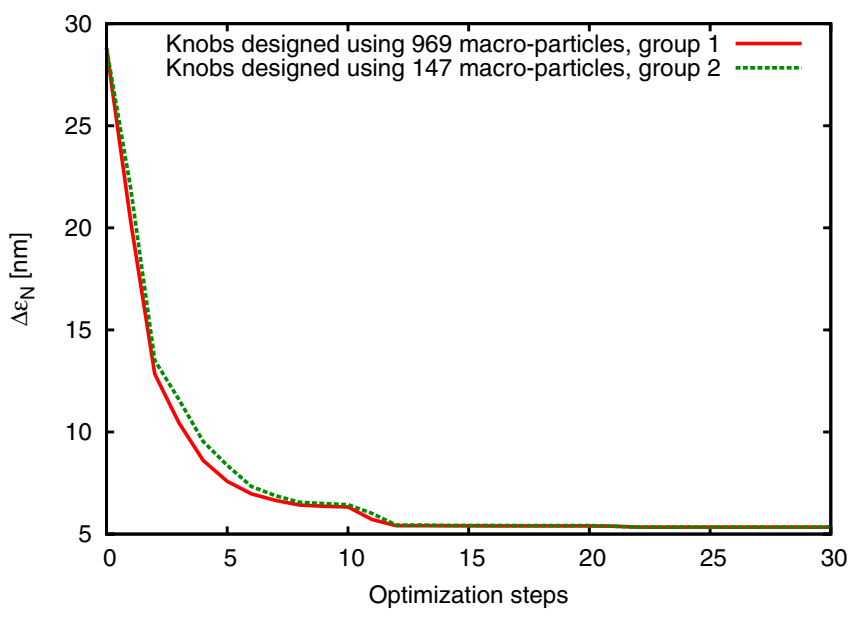

FIG. 20. Average emittance growth for 50 seeds and a beam modeled by 969 macroparticles. The performance of knobs constructed from these seeds themselves is very similar to that of knobs constructed using 50 other seeds and a 147macroparticle model. The small difference is exclusively caused by the use of a different group of seeds.

bumps. As can be seen in Fig. 21, the beam quality is drastically improved when tuning bumps are used.

In contrast to local bumps (cf. Fig. 2), the global bumps give a smooth variation of the emittance along the linac. For both types of bumps, 20 optimization steps were used, and the final emittance growth is a factor of 3 better in case of the global bumps.

\section{Tuning bumps based on quadrupole displacements}

Even though the most intuitive way to reduce the mainly wakefield induced emittance growth in CLIC is by displacements of accelerating structures, there might be other options. One possibility could, for example, be to use quadrupole displacements. All 1324 quadrupoles of the

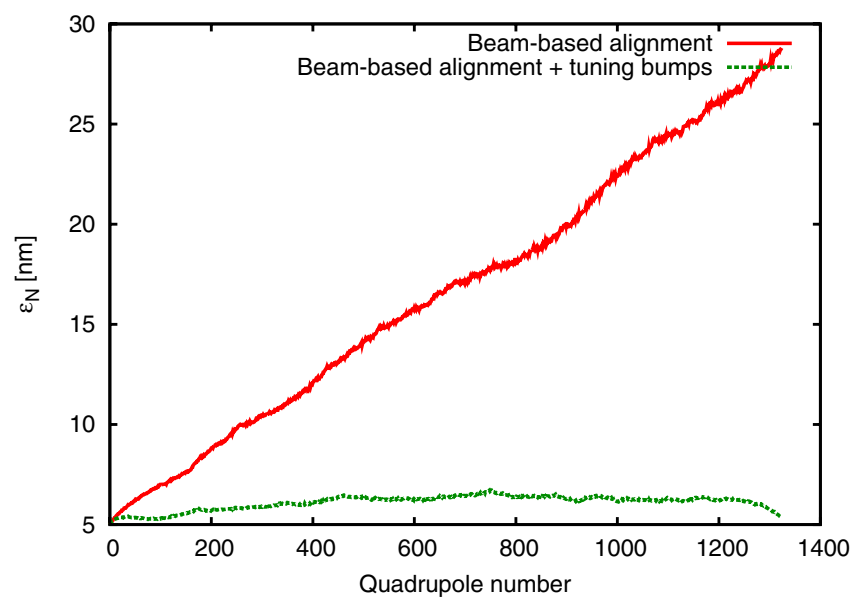

FIG. 21. Emittance along the CLIC main linac for beam-based alignment alone, compared to with the additional use of 10 optimal tuning knobs (after 20 optimization steps). 


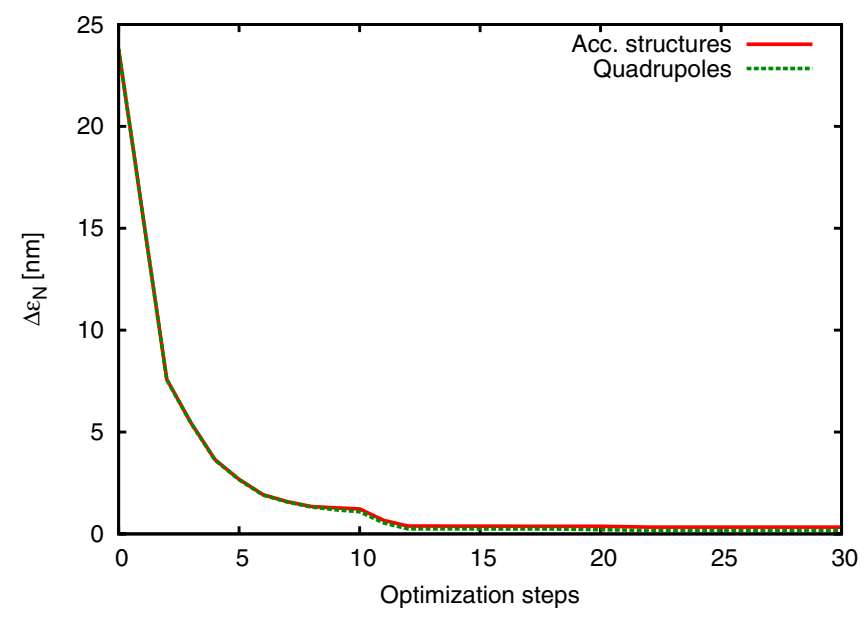

FIG. 22. Convergence of the tuning bumps based on quadrupole displacements compared to the ones based on structure displacements. The performance is nearly identical.

CLIC main linac were used to construct ten knobs using the same method as above. The performance of these knobs was almost identical to the performance of the structure knobs, see Fig. 22. The problem of using quadrupole displacements is that the performance becomes very sensitive to mover step size. Even for a step size as small as $0.1 \mu \mathrm{m}$, the bumps do no longer reduce emittance growth. By reducing the number of quadrupoles to 20 (cf. the strategy used for ILC below), the sensitivity diminishes such that ten knobs can reduce emittance growth to $6.6 \mathrm{~nm}$ for a mover step size of $0.1 \mu \mathrm{m}$ (compared to $0.55 \mathrm{~nm}$ for ideal movers). By instead using only five knobs, the lowest possible emittance growth for a $0.1 \mu \mathrm{m}$ step size is reduced to $4.6 \mathrm{~nm}$ ( $2.0 \mathrm{~nm}$ for ideal movers). Further improvement of the step size tolerance is possible but this depends on how large quadrupole displacements are acceptable. The number of quadrupoles may, for example, not be optimal. The reason for choosing 20 quadrupoles was in this case that it is the minimum number of quadrupoles required to obtain a theoretically achievable emittance growth of less than $0.5 \mathrm{~nm}$.

Bumps based on simultaneous displacements of accelerating structures and quadrupoles seem to suffer from the same sensitivity problem. A more detailed study of this might, however, be needed.

The method could of course also be used for more general knobs. Nothing prevents that additional parameters are included in the knob/seed vectors. By extending these vectors with the BPM readings at a few positions along the linac, it could be possible to construct knobs that simultaneously reduce emittance and control the beam trajectory. This would probably require using additional weights on the different coordinates in order to have the right balance between emittance reduction and trajectory control. The direct connection between the squared norm of a vector and emittance growth would, however, be lost.

\section{B. ILC}

There are a few important differences between CLIC and ILC worth mentioning. First, the use of cavities for wakefield canceling in the same fashion as for CLIC is not possible for ILC since the cavities are not foreseen to be on movers. Second, the emittance growth in ILC is mainly caused by dispersion.

During the ILC simulations, a beam consisting of 550 macroparticles was used. A more accurate beam model with a larger number of macroparticles gave identical results. Most likely fewer macroparticles could have been used without affecting the results, but even for a 550macroparticle beam the tracking through ILC is fast and a model with fewer macroparticles was never tested.

As mentioned in Sec. I, the ILC emittance growth target may be reached using beam-based alignment alone. Simulations have, however, shown that emittance tuning bumps are still useful [15]. During those earlier studies, "artificial" knobs were used without considering their actual implementation. In this case, optimal knobs based on quadrupole displacements were designed following the procedure described in Sec. III. All ILC main linac quadrupoles were used and knob vectors corresponding to the vertical displacement of each of them was calculated. By varying the number of principal knob directions and constructed knobs independently, it was found that ten principal knob directions and four knobs was a reasonable choice. According to estimates (calculated as in Sec. IIID), this would give an rms displacement of $15 \mu \mathrm{m}$ and an average remaining emittance growth of $0.05 \mathrm{~nm}$, which is close to the theoretical minimum of $0.04 \mathrm{~nm}$ obtainable along the four most important principal seed directions (cf. Fig. 10). Simulations of the knobs confirmed the excellent performance and showed that convergence is instant, see Fig. 23. While these results were obtained by correction of the same seeds as the ones used to construct the optimal knobs, very small differences were obtained when a separate set of seeds was used to set up the knobs. This was expected since the principal seed directions from two separate set of seeds had already been shown to work equally well for emittance reduction cf. Fig. 12.

A major problem of these ILC knobs is, however, that they are extremely sensitive to the minimum achievable mover step size. As mentioned in Sec. II B, this problem may be cured by reducing the number of quadrupoles. The 20 most efficient quadrupoles were chosen stepwise. One after the other, the most efficient quadrupole for emittance reduction was determined. The emittance reduction capability of a group of quadrupoles is easily calculated by SVD of the corresponding knob vectors and calculation of the $\boldsymbol{\Psi}$ matrix. The sum of the eigenvalues of this matrix gives the total achievable emittance reduction, see Sec. III D. As can be seen in Fig. 23, the performance of four knobs based on 20 quadrupoles is nearly identical to 


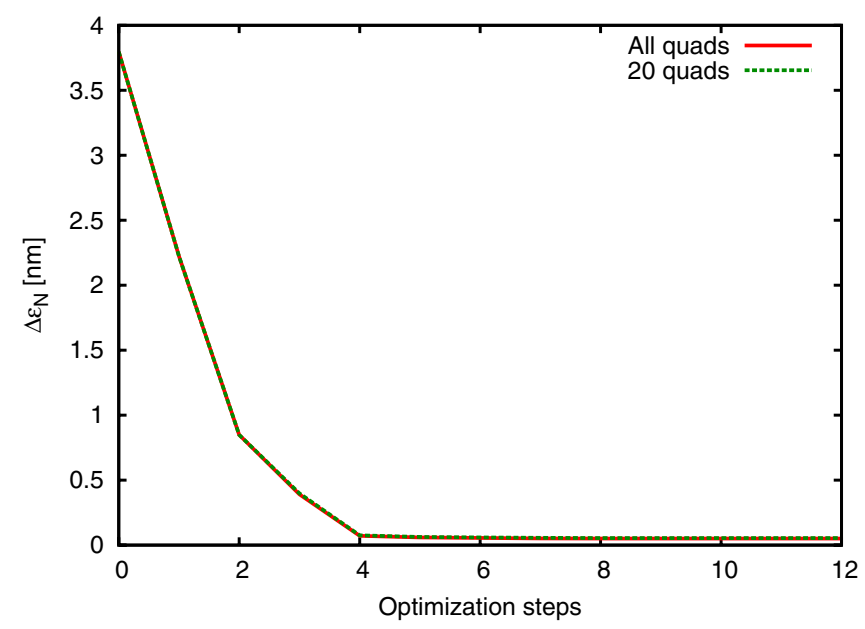

FIG. 23. Emittance growth reduction at the end of the ILC linac using four knobs. The performance is nearly identical for knobs based on all quadrupoles of the linac and knobs based on a set of 20 quadrupoles.

that of four knobs using all quadrupoles. Simulations showed that a final emittance growth of $0.054 \mathrm{~nm}$ could be obtained using 20 quadrupoles $(0.05 \mathrm{~nm}$ when all quadrupoles are used). Figure 24 shows that the sensitivity to mover step size is efficiently reduced by using a smaller number of quadrupoles. While the knobs based on all quadrupoles become useless for a step size of 0.6$0.7 \mathrm{~nm}$ or higher, the knobs using only 20 quadrupoles reduce emittance growth to $0.30 \mathrm{~nm}$ even for a step size of $1 \mu \mathrm{m}$. This improvement, however, comes hand in hand with an increase in the required quadrupole displacements. The estimated rms displacement for the 20 quadrupoles is $189 \mu \mathrm{m}$, which is also confirmed by simulations. Displacements of this order are acceptable for ILC. However, in case the requirements were stricter, the num-

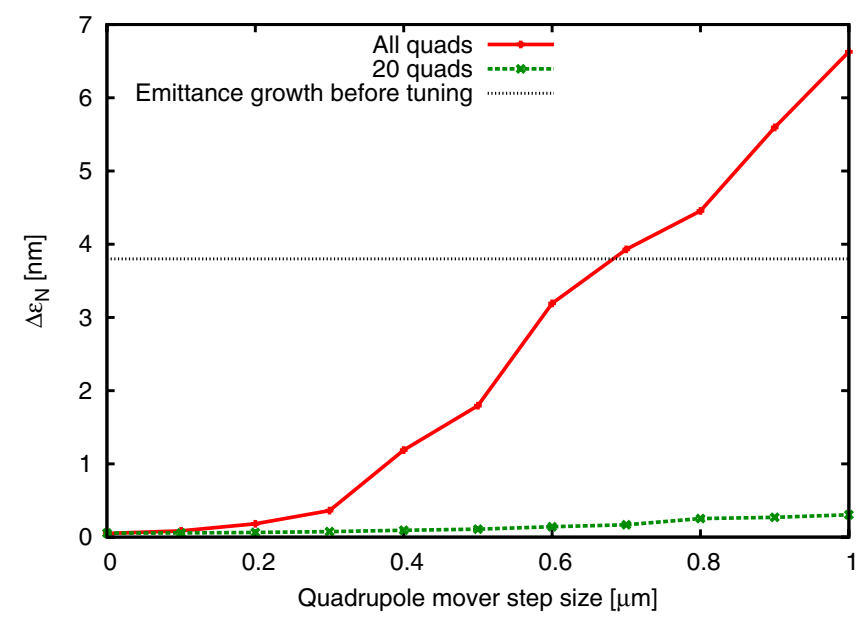

FIG. 24. Final emittance growth after tuning with four knobs assuming a finite mover step size. When all quadrupoles of ILC are used, the knobs become sensitive to step size. This can be cured by reducing the number of quadrupoles to 20 .

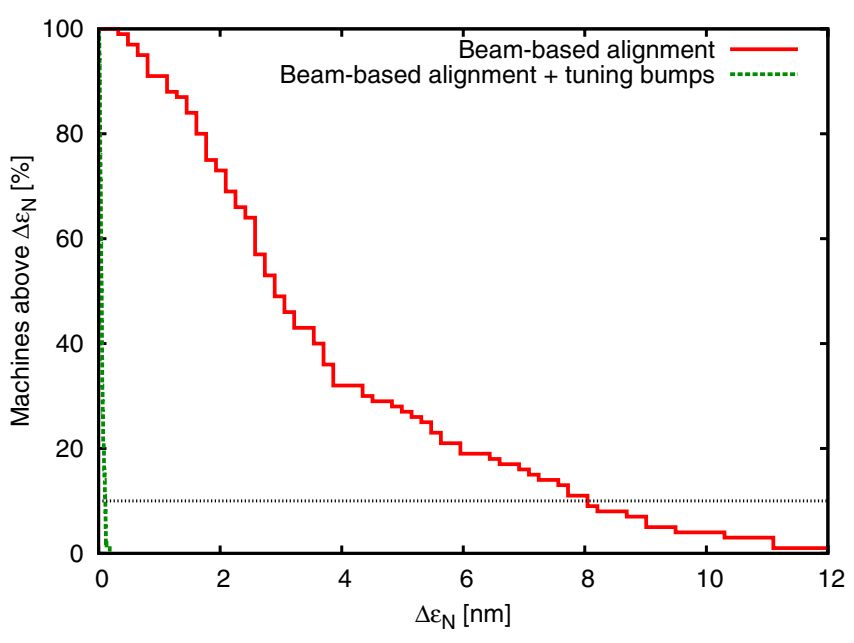

FIG. 25. Integrated histogram showing the number of machines with emittance growth higher than a certain $\Delta \epsilon_{N}$. As long as no dynamic effects are taken into account, the use of beam-based alignment alone seems to be sufficient to reach the ILC emittance target. However, the additional use of tuning bumps strongly reduce emittance growth such that $90 \%$ of all machines have a final emittance growth of less than $0.11 \mathrm{~nm}$.

ber of quadrupoles could be chosen as a trade-off between step size tolerance and acceptable displacements.

To show that the emittance target for ILC is reached, a histogram can be constructed, see Fig. 25. This histogram shows that, while beam-based alignment is barely enough to reach the target of at least $90 \%$ of all machines below $\Delta \epsilon_{N}=10 \mathrm{~nm}$, the additional use of emittance tuning bumps reduces emittance to well below the target.

In terms of final emittance reduction, the optimal knobs are only slightly better than dispersion and wakefield knobs. The convergence speed of the optimal knobs is,

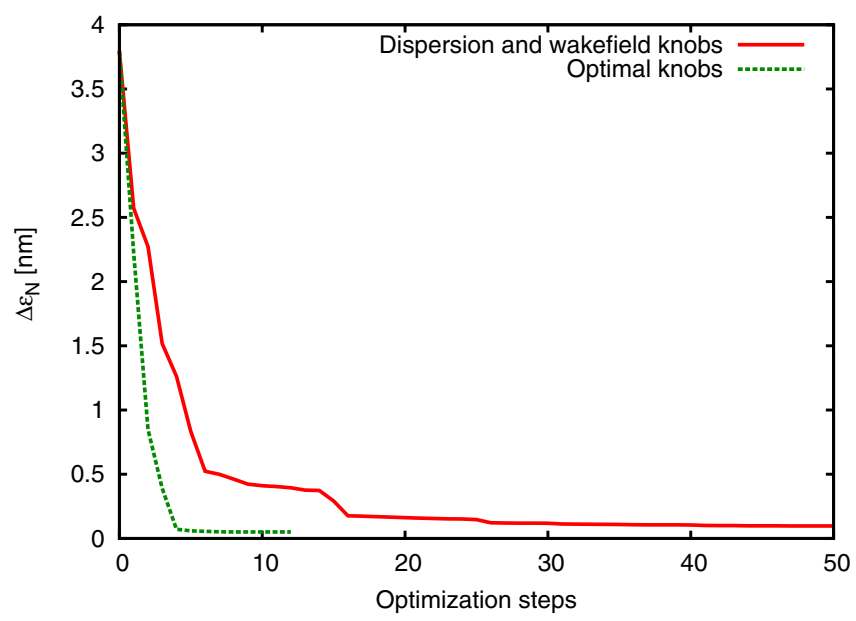

FIG. 26. Convergence of dispersion and wakefield knobs compared to convergence of optimal knobs. Even after 50 optimization steps the dispersion and wakefield knobs have not reached the low emittance obtained after four optimization steps with optimal knobs. 
however, clearly superior, see Fig. 26. The slow convergence of the dispersion and wakefield knobs is due to the fact that these knobs are not orthogonal. During these simulations, the dispersion knobs were used to control the particle coordinates directly in such a way to emulate a change in dispersion in $y$ and $y^{\prime}$. The wakefield knobs were based on cavity displacements even though no cavity movers are foreseen. In total ten such artificial knobs were used, four dispersion and six wakefield knobs. Note that the methods described in Sec. III D not only offers a way to construct optimal orthogonal knobs but also a way of constructing knobs with any desired properties. In the case of the artificial dispersion and wake field bumps described above, a realistic implementation could be obtained by replacing $\mathbf{U}_{S}$ in Eqs. (38) and (39) by the knob vectors of the artificial knobs.

\section{CONCLUSIONS}

A general strategy for the design of emittance tuning bumps has been developed. The strategy is based on singular value decomposition and can be used to diagnose large sets of seeds and knobs in order to determine the degrees of freedom that are useful for emittance reduction. A new set of optimal knobs can then be constructed as a linear combination of the old ones. These new knobs are optimal in terms of emittance reduction performance, and as a consequence of the construction method they are also noninterfering in the vicinity of the emittance minimum. It has been shown that the convergence of these noninterfering knobs is considerably better than for intuitively powerful knobs.

For CLIC, ten knobs based on displacements of 662 accelerating structures have been constructed. The fact that the knobs are nearly noninterfering makes them converge within two iterations, corresponding to 20 optimization steps. During this tuning, emittance growth is reduced from 23.8 to $0.34 \mathrm{~nm}$, improving the final emittance growth by a factor of 3 compared to using local emittance tuning bumps. The new bumps are also clearly superior to previous designs of global tuning bumps, especially in terms of convergence speed. The new bumps have been designed such that the average rms displacement of the accelerating structures does not exceed $5 \mu \mathrm{m}$. In addition, studies show that they are not particularly sensitive to limitations in minimum mover step size.

Simulations have also been carried out showing that CLIC emittance tuning bumps based on quadrupole displacements could work well. These bumps are very sensitive to mover step size. For a step size of $0.1 \mu \mathrm{m}$, emittance growth of less than $5 \mathrm{~nm}$ is, however, achievable.

For ILC, a set of four optimal knobs has been constructed. These knobs control the displacements of 20 quadrupoles, which had been identified as the 20 most efficient for emittance reduction. For ideal movers, the knobs converge in one single iteration (four optimization steps) and reduce emittance growth from 3.8 to $0.05 \mathrm{~nm}$, thus outperforming ILC bumps tested previously. Excellent emittance reduction is obtained even with a mover step size of $1 \mu \mathrm{m}$ with a final emittance growth of $0.3 \mathrm{~nm}$.

\section{ACKNOWLEDGMENTS}

This work is supported by the Commission of the European Communities under the 6th Framework Program "Structuring the European Research Area," Contract No. RIDS-011899.

[1] R. W. Assmann et al., Report No. CERN-2000-008, 2000.

[2] ILC homepage, URL http://www.linearcollider.org.

[3] F. Becker, W. Coosemans, R. Pittin, and I. Wilson, Report No. CLIC-Note-553, 2003.

[4] H. Mainaud Durand and T. Touzé, 9th International Workshop on Accelerator Alignment, 2006.

[5] G. Grzelak et al., 9th International Workshop on Accelerator Alignment, 2006.

[6] R. W. Assmann et al., CERN-2003-007, 2003.

[7] Report No. SLAC-R-606, 2003.

[8] D. Schulte, 6th European Particle Accelerator Conference : EPAC '98, Stockholm, Sweden, 1998.

[9] T. Raubenheimer and R. D. Ruth, Nucl. Instrum. Methods Phys. Res., Sect. A 302, 191 (1991).

[10] T. O. Raubenheimer and D. Schulte, for the IEEE Particle Accelerator Conference (PAC 99), New York, 1999.

[11] D. Schulte, Report No. CERN-OPEN-2003-015, 2003.

[12] P. Eliasson and D. Schulte, for the Particle Accelerator Conference (PAC 05), Knoxville, Tennessee, 2005.

[13] A. Latina, P. Eliasson, and D. Schulte, for the European Particle Accelerator Conference (EPAC 06), Edinburgh, Scotland, 2006.

[14] J.C. Smith et al. (ILC Beam Physics Group), Report No. FERMILAB-TM-2373-CD, 2007.

[15] P. Eliasson and D. Schulte, Report No. EUROTEVREPORT-2005-021, 2005.

[16] P. Eliasson, A. Latina, D. Schulte, F. Poirier, and N. Walker, for the European Particle Accelerator Conference (EPAC 06), Edinburgh, Scotland, 2006.

[17] J.T. Seeman, F. J. Decker, and I. Hsu, at the 15th International Conference on High Energy Accelerators (HEACC'92), Hamburg, Germany, 1992.

[18] C. Adolphsen, F. J. Decker, and J. T. Seeman, contributed to the 1993 Particle Accelerator Conference (PAC 93), Washington, DC, 1993.

[19] E. T. D'Amico, G. Guignard, N. Leros, and D. Schulte, at the IEEE Particle Accelerator Conference (PAC2001), Chicago, Illinois, 2001.

[20] G. H. Golub and C.F. Van Loan, Matrix Computations (The Johns Hopkins University Press, Baltimore, Maryland, 1996), 3rd ed.

[21] W.H. Press, B.P. Flannery, S. A. Teukolsky, and W.T. Vetterling, Numerical Recipes: The Art of Scientific Computing (Cambridge University Press, Cambridge, England, 1992), 2nd ed., ISBN 0-521-43064-X.

[22] OCTAVE homepage, URL http://www.octave.org. 